EPJ manuscript No.

(will be inserted by the editor)

\title{
Energy landscapes, lowest gaps, and susceptibility of elastic manifolds at zero temperature
}

\author{
E. T. Seppälä and M. J. Alava \\ Helsinki University of Technology, Laboratory of Physics, P.O.Box 1100, FIN-02015 HUT, Finland
}

Received: December 12, 2000 / Revised version: April 11, 2001

\begin{abstract}
We study the effect of an external field on $(1+1)$ and $(2+1)$ dimensional elastic manifolds, at zero temperature and with random bond disorder. Due to the glassy energy landscape the configuration of a manifold changes often in abrupt, "first order" -type of large jumps when the field is applied. First the scaling behavior of the energy gap between the global energy minimum and the next lowest minimum of the manifold is considered, by employing exact ground state calculations and an extreme statistics argument. The scaling has a logarithmic prefactor originating from the number of the minima in the landscape, and reads $\Delta E_{1} \sim L^{\theta}\left[\ln \left(L_{z} L^{-\zeta}\right)\right]^{-1 / 2}$, where $\zeta$ is the roughness exponent and $\theta$ is the energy fluctuation exponent of the manifold, $L$ is the linear size of the manifold, and $L_{z}$ is the system height. The gap scaling is extended to the case of a finite external field and yields for the susceptibility of the manifolds $\chi_{\text {tot }} \sim L^{2 D+1-\theta}[(1-\zeta) \ln (L)]^{1 / 2}$. We also present a mean field argument for the finite size scaling of the first jump field, $h_{1} \sim L^{d-\theta}$. The implications to wetting in random systems, to finite-temperature behavior and the relation to Kardar-Parisi-Zhang non-equilibrium surface growth are discussed.
\end{abstract}

PACS. 75.50.Lk Spin glasses and other random magnets - 05.70.Np Interface and surface thermodynamics -68.08.Bc Wetting - 74.60.G Flux pinning, flux creep, and flux-line lattice dynamics

\section{Introduction}

In this paper we study zero-temperature or ground state elastic manifolds that are roughened by bulk disorder, in the presence of an external field. Such objects are relevant in many contexts of condensed matter and statistical physics [1,2. The essential point here is a competition between the elasticity, which prefers flat manifolds, and the disorder, which induces wandering in order to take advantage of the low energy regions in the system. This leads to a glassy, complicated energy landscape and the fact that the quenched randomness dominates thermal effects at low temperatures. In two embedding dimensions (2D) such manifolds are under the name directed polymers (DP) [3, 4, 5] particularly interesting through their connection to the celebrated Kardar-Parisi-Zhang (KPZ) equation of nonlinear surface growth. Directed polymers have an experimental realization as vortex-lines in granular superconductors [6]. In higher dimensions elastic manifolds may be best considered as domain walls (DW) in ferromagnets with quenched impurities. Elastic manifolds have also other connections to charged density waves, the sine-Gordon model with disorder, random substrate problems, and vortex lattices, to name but a few [6, 7, 8].

Let us start by introducing the classical spin-half random Ising Hamiltonian

$$
\mathcal{H}=-\sum_{\langle i j\rangle} J_{i j} S_{i} S_{j}-\sum_{i} H_{i} S_{i}
$$

where $J_{i j}$ is the coupling constant between the nearestneighbor spins $S_{i}$ and $S_{j}$, and $H_{i}$ is a field assigned to each spin. To this system we apply antiperiodic or domain wall -enforcing boundary conditions in one direction. The spins in the opposite boundaries, let us define in $z$-direction, $z=$ 0 and $z=L_{z}$ are forced to be up and down, respectively. In the case of ferromagnetic random bond (RB) Ising systems one has $J_{i j} \geq 0$ and $H_{i}=0$. In the minimum energy state the spins prefer to align on each side of the induced domain wall. When $J_{i j} \lessgtr 0$ the spins become frustrated and the task to find the ground state (GS) structure is most often related to spin glass physics [9, 10,11]. On the other hand, when for simplicity $J_{i j}=$ const. $=J>0$, and $H_{i} \lessgtr 0$ one arrives at random field (RF) Ising systems. The random field Ising model (RFIM) has an experimental realization as a diluted antiferromagnet in a field. In the RFIM the ferromagnetic couplings compete with the random field contribution, which prefers in the ground state to have the spins to be oriented towards the field assigned to them.

Here we concentrate mainly on the random bond Ising Hamiltonian, i.e., $J_{i j}>0$ and $H=0$, and in some special cases extend the discussion to RF domain walls as well. In the simplest case the spins are located in a square lattice, in $d=2$, or a cube in $d=3$, so that the lattice orientation is in the $\{10\}$ and $\{100\}$ directions, respectively. The elastic manifold is the interface, with the dimension $D=d-1$, which divides the system in two parts of up and down spins. At $T=0$ the problem of finding the ground 
state domain wall, which minimizes the path consisting of unsatisfied bonds between the spins on opposite sides of the domain wall becomes "global optimization". In our case the displacement field is one-dimensional, $n=1$, but one can certainly think about generalizations so that the total dimension of a system $d=(D+n)$, where $n \geq 1$ is the dimension in which the manifold is able to fluctuate.

The continuum Hamiltonian of elastic manifolds with an external field and $n=1$ may be written as

$$
\mathcal{H}=\int\left[\frac{\Gamma}{2}\{\nabla z(\mathbf{x})\}^{2}+V_{r}\{\mathbf{x}, z(\mathbf{x})\}+h\{z(\mathbf{x})\}\right] \mathrm{d}^{D} \mathbf{x} .
$$

The elastic energy is proportional to the area of the interface given by the first term, and $\Gamma$ is the surface stiffness of the interface. The second term of the integrand comes from the random potential, and the last term accounts for the potential caused by the external field. The use of random bond disorder means that the random potential is delta-point correlated, i.e., $\left\langle V_{r}(\mathbf{x}, z) V_{r}\left(\mathbf{x}^{\prime}, z^{\prime}\right)\right\rangle=2 \mathcal{D} \delta(\mathbf{x}-$ $\left.\mathbf{x}^{\prime}\right) \delta\left(z-z^{\prime}\right)$. In the random field case $\left\langle V_{r}(\mathbf{x}, z) V_{r}\left(\mathbf{x}^{\prime}, z^{\prime}\right)\right\rangle \sim$ $\delta\left(\mathbf{x}-\mathbf{x}^{\prime}\right)\left(z-z^{\prime}\right)$. The Hamiltonian (2) is also applicable to wetting in a three phase system, where two of the phases are separated by an interface in a random bulk [12, 13, 14]. In that case $h(z)$ is equivalent to the chemical potential, which tries to bind the interface to the wall, and competes with the random potential, in the presence of which the interface tends to wander in the low energy regions of the system.

Below the upper critical dimension the geometric behavior of elastic manifolds is characterized by the spatial fluctuations. For the mean-square fluctuations one has

$$
w^{2}=\left\langle[z(\mathbf{x})-\overline{z(\mathbf{x})}]^{2}\right\rangle \sim L^{2 \zeta}
$$

where $z(\mathbf{x})$ is the height of the interface with the mean $\bar{z}$, $\mathbf{x}$ is the $D=d-n$ dimensional internal coordinate of the manifold, $L$ is the linear size of the system, and $\zeta$ is the corresponding roughness exponent. At low temperatures in $(D+n)=(1+1)$ dimensions with $\mathrm{RB}$ disorder, i.e., when one actually considers a directed polymer in random media [3,4], the roughness exponent is calculated exactly via the $\mathrm{KPZ}$ formalism to be $\zeta=2 / 3$. In higher manifold dimensions $D$ with $n=1$ the functional renormalization group (FRG) calculations give the approximatively values $\zeta \simeq 0.208(4-D)$ for $\mathrm{RB}$ disorder and $\zeta=(4-D) / 3$ for RF disorder [1, 15]. The expression for $\zeta$ tells also that the upper critical dimension for the elastic manifold is $D_{u}=4$. For manifolds with varying $n$ and $D$ Balents and D. Fisher have derived using $\mathrm{FRG} \zeta \simeq[(4-D) /(n+$ 4) $]\left\{1+(1 / 4 e) 2^{-[(n+2) / 2]}\left[(n+2)^{2} /(n+4)\right][1-\ldots]\right\}$ 16]. At zero temperature the total average energy $\bar{E}$ of an elastic manifold equals its free energy and grows linearly with the system size $L^{D}$ and its fluctuations scale for all $n$ as $\Delta E=\left\langle(E-\bar{E})^{2}\right\rangle^{1 / 2} \sim L^{\theta}$, where 17]

$$
\theta=2 \zeta+D-2
$$

is the first non-analytic correction to the energy. The same hyperscaling law holds for RF manifolds, too, in $D>1$
[18]. Having a positive $\theta$ implies that the temperature is an irrelevant variable in the renormalization group (RG) sense and the $T=0$ fixed point dominates. For $D=1$, $n>2$ there exists a $T_{c}$, and $T=0$ fixed point dominates only below $T_{c}$, likewise always for $n \leq 2 \sqrt{19}$. At the randomness dominated pinned phase the temperature is "dangerously" irrelevant, which means that in RG calculations the interesting correlation functions cannot be obtained by setting $T$ to zero. Above $T_{c}$ the fluctuations become random walk -like with $\zeta=1 / 2$ and $\theta=0$. For $D=1$ with $n>1$ there is no exact result existing for the roughness exponent below $T_{c}$, and hence whether $\zeta \rightarrow 1 / 2$ and $\theta \rightarrow 0$ for a finite $n_{c}$ is still an open question - that is, what is the upper critical dimension of the KPZ equation.

Elastic manifolds self-average in the sense that the intensive fluctuations of the roughness and the energy decrease with system size. However, in this paper we will show that, due to the glassiness of the energy landscape, for example the behavior of the mean position of a typical example of a manifold $\overline{z(\mathbf{x})}$ does not coincide with the disorder average, here denoted by \langle\rangle , over many realizations with different random configurations. Introducing the external field to a random system induces often drastic changes and one experimental possibility is to study the ground state behavior e.g. by measuring the susceptibility. In disordered superconductors the external field is due to the current density $j$, which drives the vortex lines.

Here we study the elastic manifolds in an external field while being especially interested in the information it gives about the energy landscape of the random system [20], which is closely related to the susceptibility. The external field is applied to the coupling constants [see Eq. (2)] so that $J_{\perp}(z)=J_{\text {random }}+h(z)$, where $J_{\perp}$ are ones in the $z$-direction and $h$ is the amplitude of $h(z)$, the strength of the external field. Since we have the field potential linear in height, $h(z)=h z$, in ferromagnetic random systems with a domain wall the external field $h(z)$ may be transformed to a constant external field term $-H \sum_{i} S_{i}$ in the Hamiltonian (1). Our results are a systematic extension of early numerical work by Mezárd and relate to more recent discussion of the energy landscapes of directed polymers by Hwa and D. Fisher [21,22]. Due to the glassiness of the energy landscape the position changes in "first order type" large jumps, at sample-dependent values of the external field. The second scenario in which the perturbations take place locally via "droplet"-like excitations is ruled out by a scaling argument and numerical results.

We start by studying a specific case where the number of valleys is fixed to a constant. We first consider the case without the external field, and find the scaling behavior of the energy difference of the global energy minimum and the next lowest minimum of a manifold numerically and derive it also from an extreme statistics argument. The scaling of the gap does not only depend on the energy fluctuation scale, but has in addition a logarithmic factor dependent on the number of the low energy minima. The gap scaling is extended to the finite external field case, to derive the susceptibility of the manifolds under the assumption that the zero-field energy landscape is still 
relevant. The corresponding numerics allows us also to deduce the effective gap probability distribution without any a priori assumptions. We then proceed by constructing a mean field argument for the finite size scaling of the first jump field in a more general case. This agrees with "grandcanonical" numerics in which the manifold is allowed to have an arbitrary ground state position in the system: the number of valleys in the energy landscape fluctuates and the important physics is caught by the simple scaling considerations. Finally, we discuss wetting in random systems in the light of our results, and compare with the necklace theory of M. Fisher and Lipowsky that applies in the limit of large fields. We also consider finite temperatures, and relate the physics at finite external fields to the Kardar-Parisi-Zhang growth problem through the arrival time mapping. The implications of our results in that case concern the two first arrival times and their difference of an interface to a prefixed height. Note that we have published short accounts of some of the work contained here earlier 23, 24.

The paper is organized so that it starts with a short review of the thermodynamic behavior of the interfaces in random media at zero temperature in the presence of an applied field, and of the folklore concerning energy landscapes, in Section 2. Section 3 introduces the mean-field level behavior of the interface when the external field is applied. The analytical probability distribution of the first jump field is derived and its relation to the susceptibility is discussed in Section 1 . In the section also the lowest energy gaps between the local energy minima and the global minimum are derived from extreme statistics arguments. The numerical method of calculating the first jump field $h_{1}$ of an interface in a fixed height is introduced in Section 5.1. Section 5.2 contains the numerical results of the first jump field in $(1+1)$ and $(2+1)$ dimensions with the corresponding jump geometry statistics as well as the energy gaps of the first lowest energy minima. The results are compared with the analytical arguments presented in the previous section. In Section 6 a mean field argument for the first jump field of an interface which lies originally in an arbitrary height is derived and compared with the numerical data and the evolution of the jump size distributions is studied. As an application for the finite field case the wetting phenomenon is considered and the corresponding wetting exponents are numerically studied in Section 0 . The arguments presented in the paper are discussed from the viewpoint of interface behavior at low but finite temperatures in Section 8.1 and in the context of first arrival times in KPZ growth in Section 8.2. The paper is finished with conclusions in Section 9 .

\section{Thermodynamic considerations}

The interesting behavior arises since in the low temperature phase e.g. directed polymers are found to have anomalous fluctuations resulting from the regions of the random potential with almost degenerate energy minima, which are separated by large energy barriers [19, 21, 22, These spatially large-scale low-energy excitations are rare, but are expected to dominate the thermodynamic properties and cause large variations in the structural properties at low temperatures. We will use the arguments in the next section in order to study the movement of the manifolds in equilibrium when an external field is applied. Hwa and D. Fisher [22] derived that for a polymer fixed at one end a small energy excitation with a transverse scale $\Delta\left(\simeq l^{\zeta}\right.$, where $l$ is the linear length of the excitation), scales as $\Delta^{\theta / \zeta}$ and gives rise to large sample-to-sample variations in the two-point correlation function and dominates the disorder averages. The probability distribution for the sizes of the excitations was found to be a power-law from the normalization of "density of states" of small energy excitations $W \sim \Delta^{-n} l^{-\theta} \sim 1 / \Delta^{n+2-1 / \zeta}$, since $\theta=2 \zeta-1$, Eq. (4). This is in contrast to the high temperature phase one, where $W$ is Gaussian. For the finite field case Hwa and D. Fisher argued that the power-law distribution for the excitations is due to the statistical tilt symmetry, which means that the random part of the new potential of the polymer is statistically the same as the old one, when the polymer is excited by an applied field. In Ref. [21] Mézard showed numerically that the energy difference of two copies of the polymer in the same realization of the random potential scales as $L^{\theta}$, where $\theta=1 / 3$ in $d=2$. He also showed that the ground state configuration of a polymer, which is fixed at one end and applied with an external field $h$ to the end point of the polymer, i.e., $h\{z(\mathbf{x})\}=h z \delta(x-L)$ in Hamiltonian (2), changes abruptly with a distance $L^{\zeta}, \zeta=2 / 3$, when the field is increased by $L^{\theta}$. Calculating the variance of the end point of the polymer $\operatorname{var}(z) \sim\left\langle z^{2}\right\rangle-\langle z\rangle^{2}$ Mézard found that typically it is zero, i.e., the ground state configuration does not change. With the probability $L^{-\theta}$, there is a sample for which $h=0$ is the critical value of $h$, and the configuration changes with a large difference compared to the

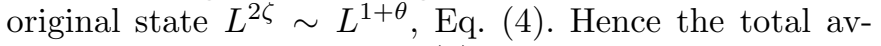
erage variance becomes $\operatorname{var}(z)_{\text {ave }} \sim L$. In this paper we generalize such studies as discussed later.

The physics of DP's have been shown by Parisi to obey weakly broken replica symmetry [25], a "baby-spin glass" phenomenon. This means that the replica symmetric solution of a DP is degenerate with the solution with the broken replica symmetry. This is also evidence for an energy landscape with several, far away from each other, nearly degenerate local minima. Mézard and Bouchaud studied later the connection between extreme statistics and one step replica symmetry breaking in the random energy model (REM) [26, in a finite, one-dimensional form [27. They computed using extreme statistics the probability distribution of the minimum of all the energies $\frac{\gamma}{2} x^{2}+E(x)$, where $E(x)$ are random and follow a suitable probability distribution so that its tails decay faster than any power-law, e.g. Gaussian. This can be seen as a toy model for an interface in a random media, in which case the quadratic part plays the role of the elasticity. The minimum of the total energy is easily seen to be distributed according to the Gumbel distribution 28 of extreme statistics, and likewise for the position of the interface the shape of the probability distribution to be ap- 
proximately Gaussian. In Section 1 we will use extreme statistics to study the scaling of the lowest minima and energy gaps in elastic manifolds in a situation analogous to that of Bouchaud and Mézard.

The response of the manifolds pinned by quenched impurities to perturbations was discussed in terms of thermodynamic functions by Shapir [29], including the susceptibility of the manifolds. The formal definition of the susceptibility for a $D$ dimensional manifold in a $d$ dimensional embedding space reads:

$$
\chi=\lim _{h \rightarrow 0+}\left\langle\frac{\partial m}{\partial h}\right\rangle,
$$

where the change in the magnetization of the whole $d$ dimensional system is calculated in the limit of the vanishing external field from the positive side and the brackets imply disorder average. In Section 5.2 we derive the susceptibility based on energy landscape arguments. By assuming smooth, analytic behavior in the manifolds' thermodynamic functions, Shapir found that the susceptibility (for a surface of dimension $D$ ) is proportional to the displacement of the manifold in the limit of small field, which is applied uniformly to the whole manifold, and $\chi_{D} \sim$ $d^{2} E / d h^{2} \sim L^{\theta+2 \tilde{\alpha}}$. $\tilde{\alpha}$ results from an argument concerning the energy gap and the external field: $\Delta E \sim h L^{\zeta} L^{D}$, where the external field couples to a droplet of size $L^{\zeta}$ and the area of the manifold is $L^{D}$. This should be equal to the energy difference: $\Delta E \sim L^{\theta+\tilde{\alpha}}$, and thus $\tilde{\alpha}=2-\zeta$, since $\theta=2 \zeta+D-2$. Hence the susceptibility was derived to be $\chi_{D} \sim L^{D+2}$ and the susceptibility per unit hyper-surface vary as $L^{2}$ for a manifold of scale $L$. This surprisingly is independent of the type of the pinning randomness.

The landscapes of DP's have also been studied by Jögi and Sornette [30] by moving both end points step by step (with periodic boundary conditions) from $(0, z)$ and $(L, z)$ to $(0, z+1)$ and $(L, z+1)$, and finding the ground state at each step. They studied the sizes of the avalanches as in self-organized systems, i.e., the areas the polymer covers when moving to the next position. They found a powerlaw for the avalanche sizes $P(S) d S \sim S^{-(1+m)} d S$, where $m=2 / 5$, up to the maximum size $S_{\max } \sim L^{5 / 3}$. $S_{\max }$ is actually the size of the largest fluctuation of the polymer in such a controlled movement, i.e., $L \times L^{\zeta}$. The authors did not however study the energy variations of the manifold during the "dragging process".

In the limit of a large $h$ the Hamiltonian of Eq. (2) is applicable to (complete) wetting interface in random systems with three phases. In this case the manifold is the interface between i) non-wet and ii) wet phases near iii) a hard wall at $z=0$. This problem was studied by mean-field arguments by first Lipowsky and M. Fisher and later elaborated numerically 12,13. The choice of wetting potential that corresponds to the linear field used here is called in the wetting literature the weak-fluctuation regime (WFL). In the WFL regime one can derive the wetting exponent $\psi$ by a Flory argument, since the mean distance of the interface from the inert wall $\bar{z}$ is of the order of the interface transverse fluctuations $\xi_{\perp}$, see Fig. 1, i.e., the field is strong enough to bind the interface to the wall.
The Hamiltonian can now be minimized by estimating the total potential to be $V_{t o t}=V_{f l}(\bar{z})+V_{W}(\bar{z}) . V_{W} \sim h z$ is the wetting potential induced by the external field $h$. The fluctuation-induced potential $V_{f l}$ is the potential of the free interface $V_{e l}+V_{r}=\frac{\Gamma}{2}\{\nabla z(\mathbf{x})\}^{2}+V_{r}(\mathbf{x}, z)$, which gives, when minimized, $\xi_{\perp} \simeq \xi_{\|}^{\zeta}$. The elastic energy term $V_{e l}$ gives the scaling of $V_{f l}$ with respect to the correlation lengths, and expanding the square-term of the gradient of the height gives const ${ }_{1}+$ const $_{2}\left|\frac{\xi_{\perp}}{\xi_{\|}}\right|^{2}+$ higher order terms. So, $V_{f l} \sim \xi_{\perp}^{-\tau}$, where the decay exponent $\tau=(2-2 \zeta) / \zeta$ defines the scaling between the pinning and elastic energies. Minimizing $V_{\text {tot }}$ in WFL, where $\bar{z} \simeq \xi_{\perp}$ and is much larger than a lattice constant, gives

$$
\bar{z} \sim h^{-1 /(1+\tau)} \sim h^{-\psi} .
$$

Thus the wetting-exponent $\psi=1 /(1+\tau)$ becomes

$$
\psi=\frac{\zeta}{2-\zeta}
$$

Huang et al. [31, 13] did numerical calculations for directed polymers at zero temperature using transfer-matrix techniques for slab geometries, $L \gg L_{z}$, where $L$ is the length of the polymer, and $L_{z}$ is the height of the system, confirming roughly the expected exponent $\psi \simeq 0.5$. Our results, presented in Section 7 are in line with the expectations in $(1+1)$ and we also present the first studies in $(2+1)$ dimensions.

\section{Glassy energy landscapes: length scale of perturbations}

We next employ a version of the scaling arguments by Hwa and Fisher, and Mézard for the next optimal position of the directed polymer. The goal is to investigate the preferred length scale of the change that takes place, i.e., the size of the "droplet" created with the field. The numerical calculations presented in this paper are done such a way, that first the ground state is searched and the configuration stored. After that the external field is applied and the energy is minimized again. If there is a change in the configuration, the difference in the mean height of the manifold, the so called "jump size" is analyzed. Most of the studies are done in isotropic systems, i.e., the height $L_{z}$ of the system is of the order of the linear length of the manifold, $L_{z} \propto L$. One finds that the behavior of the polymer has a first order character: the optimal length scale is such that the whole configuration changes, and this holds for higher dimensional manifolds, too.

Hwa and Fisher defined the next optimal position of the DP fixed at one end and with displacement $\Delta$ to scale as $\Delta \sim L^{\zeta}$, where $L$ is the length of the polymer. Now one assumes that the energy gaps between two copies of the polymers with overlap, and energies $E_{0}$ and $E_{1}$, grows as $E_{1}-E_{0} \sim L^{\theta}$, i.e., is just energy fluctuation exponent of a polymer (as demonstrated by Mézard numerically). Then 
as a generalization with $n=1$ it follows that $E_{1}-E_{0} \sim$ $\Delta^{\theta / \zeta}$. The external field has a contribution for the energy differences of interfaces with the dimension $D E_{1}-E_{0} \sim$ $h L^{D} \Delta \sim h \Delta^{1+D / \zeta}$. Assuming that this difference balances the gap, and using the hyperscaling law for the roughness and energy fluctuation exponents $\theta=2 \zeta+D-2$, we get

$$
h \sim \Delta^{\bar{\alpha}}=\Delta^{(\zeta-2) / \zeta}
$$

The exponent $\bar{\alpha}$ is negative assuming that the roughness exponent is below two, which is of course satisfied in the case of both RF and RB domain walls. Hence, the smaller the field the larger the excitations and thus large excitations are the preferred ones, at least below the upper critical dimension. Besides RB systems Eq. (8) works only for RF systems with a ferromagnetic bulk (among others, the relation $E_{1}-E_{0} \sim L^{\theta}$ has not been demonstrated to hold for $\mathrm{RF}$ interfaces). If the bulk of a RF magnet is paramagnetic, the stiffness of DW's is expected vanish on large enough length scales, translational invariance is broken, and $\Delta E=E_{1}-E_{0}$ does not scale. The scaling relation (8) should hold also for general $n$. One should note in the case $h$ is applied in one direction but the droplet may extend in $n$ dimensions, in order to Eq. (8) to hold, also the projection of the droplet in the applied field direction has to have the scaling as $\Delta^{\theta / \zeta}$.

In Fig. 2 it is shown what happens for two different random realizations of DPs, when a perturbing external field is applied. Fig. 2(a) shows the mean height of the polymer normalized by its original height $\bar{z} / \bar{z}_{0}$, when the external field is increased. The first realization $1^{\circ}$ shows a large jump of a size half of the height of its original position at first jump field $h_{1}=8 \times 10^{-5}$. In Fig. 2(b) the two positions of $1^{\circ}$, before and after the first jump, $z_{0}(x)$ and $z_{1}(x)$, are shown. The other scenario would be that the directed polymer would continuously undergo small geometric adjustments and get meanwhile bound to the wall, $z=0$. An example of such a droplet, is shown in Fig. 2(b) as the second realization $2^{\circ}$, see also Fig. 1. However, a further field increase, demonstrated in Fig. 2(a) for $2^{\circ}$, leads to a global jump too. This leads to a picture in which assuming a starting position far enough from the system boundary, a finite number of large jumps exists from the original position $z_{0}(\mathbf{x})$ to the positions $z_{1}(\mathbf{x}), z_{2}(\mathbf{x}), \ldots, z_{n}(\mathbf{x}), \ldots$, closer and closer to the wall, i.e., $z_{0}(\mathbf{x})>z_{1}(\mathbf{x})>z_{2}(\mathbf{x})>\ldots>z_{n}(\mathbf{x})>0$.

The mean jump length is defined to be $\Delta z_{n}=\bar{z}_{n-1}-$ $\bar{z}_{n}$, and the jumps take place at the fields $h_{1}, h_{2}, \ldots, h_{n}, \ldots$. The field value $h_{1}$ corresponds the jump from $z_{0}(\mathbf{x})$ to $z_{1}(\mathbf{x})$. Finally, after a finite number of jumps, with the interface being in the proximity of the wall, around $h \simeq$ $2 \times 10^{-2}$ in Fig. 2(a), see also Fig. 1, the interface evolves inside the last valley next to the wall continuously, and the wetting behavior, Eqs. (6) and (7), applies. We discuss this picture of consequent jumps before the wetting regime in Section 6 together with the numerical results.

The global changes (large jumps) induce finite changes in the magnetization $(m)$, and are reminiscent of first order phase transitions. Note that the field $h_{1}$ of the first change or jump obeys for any particular ensemble a prob- ability distribution, and thus a co-existence follows between systems that have undergone a jump or change in $m$ and those that are still in the original state. This "transition" is a result of level-crossing between the valleys in the energy landscape for the interface, sketched in Fig. 3 . To change between the geometrically separated minima, an external field is applied, which at jumps plays the role of a latent heat. Originally the interface lies at height $\bar{z}_{0}$ and has an energy $E_{0}$. When the field is applied interface's energy increases by $h L^{D \bar{z}_{0}}$ and at $h_{1}$ the interface jumps to $\bar{z}_{1}<\bar{z}_{0}$ having energy $E\left(h_{1}\right)=E_{1}+h_{1} L^{D} \bar{z}_{1}=$ $E_{0}+h_{1} L^{D} \bar{z}_{0}$, where $E_{1}$ is the energy of the interface at $z_{1}$ without the field. Similar behavior takes place at $h_{n}, n=2,3, \ldots$, when the interface moves from $\bar{z}_{n-1}$ to $\bar{z}_{n}<\bar{z}_{n-1}$, until the cross-over to the wetting regime.

In the thermodynamic (TD) limit, i.e., for very large systems, when $L, L_{z} \rightarrow \infty$, the interface may originally be located anywhere in the system. Assuming that the roughness exponent $\zeta$ does not define only the width of the manifold, but also the width of the minimum energy valley, i.e., $L^{\zeta}$ is the only relevant length scale in the transverse direction, gives that the manifold should have $N_{z} \sim L_{z} / L^{\zeta}$ minima from which to choose the global minimum position. For $\zeta<1$ the number of the minima grows with system size, if the geometry is kept isomorphic, $L_{z} \propto L$. The requirement $\zeta<1$ holds for all RB interfaces; and for $\mathrm{RF}$ ones with ferromagnetic bulk, when $D>1$. In the special case that the local minimum closest to the wall coincides with the global minimum, the wetting regime is entered at once without any jumps. The prediction of Eq. (8) that large scale excitations should dominate is an asymptotic one, and for finite system sizes one may find also small excitations, which are less costly in energy (Fig. 2). However, the fraction of these cases decreases with system size, see Fig. It It depicts in $d=2$ up to $L^{2}=1000^{2}$, with at least $N=3000$ realizations per data point, the probability of finding a non-zero overlap $q$ between the interfaces before and after the first jump. $q$ is the disorder average of the fraction of the samples, which have overlap between the cases before and after the jump, i.e., for at least one $x z_{0}(x)=z_{1}(x)$. The probability goes as $q \simeq 0.56 L^{-0.23 \pm 0.01}$, and confirms the expected behavior that for isotropic systems in the $L \rightarrow \infty$ limit the first jump is always without an overlap, i.e., a macroscopic one. A first guess would give that $q \sim 1 / N_{z} \sim L^{\zeta-1}$. A similar trend seems to exist in $(2+1)$ dimensions, too, but our statistics is not good enough to determine the exponent of $q$ reliably. This is since $q$ is averaged over a binary distribution (overlapping and non-overlapping jumps), and thus very large ensembles would be needed.

\section{Extreme statistics arguments}

Next we compute the field $h_{1}$ and the susceptibility, Eq. (5), by taking into accont the "co-existence" of the original ground state and the state after the jump. Assuming that the relevant process in the response of a domain wall is the large-scale jump, the susceptibility per spin of a system with a domain wall, Eq. (5), may be written in the 
form

$$
\chi=\lim _{h \rightarrow 0+}\left\langle\frac{\Delta m(h)}{\Delta h}\right\rangle \simeq\left\langle\frac{\Delta z_{1}}{L_{z}}\right\rangle \lim _{h \rightarrow 0+} P\left(h_{1}\right),
$$

where $P\left(h_{1}\right)$ is the probability distribution of the first jump fields with the corresponding first jump size $\Delta z_{1}$, and the magnetization $m(h) \sim z(h) / L_{z}$. The limit $h \rightarrow$ $0+$ is taken over the probability of having a jump, and thus the susceptibility will reflect the co-existence phenomenon discussed above. One uses also a plausible assumption, found to be true in the numerics, that the change in the interface does not depend on the threshold field $h_{1}$. A further simplification is obtained by assuming (this will be shown numerically later) that the jumps have an invariant size distribution, independent of $L$ and $L_{z}$, after a normalization with $L_{z}$. This gives $\left\langle\Delta z_{1}\right\rangle \sim L_{z}$. Hence the finite size scaling of the susceptibility per spin depends only on the probability distribution of the first jump field in the limit of the vanishing external field, and in particular on the "rare events" measured by such a distribution. The dependence of $h_{1}$ on the anomalous scaling is found to be true not only for directed polymers but also for the higher dimensional case. Such behavior is contrary to Shapir's work that assumed smooth, analytic thermodynamic functions and it is thus no surprise that the scaling of the susceptibility, derived in detail in Section 5.2, differs from that.

Next we first derive the distribution for the first jump field and its relation to the lowest energy gap probability distributions. Then the probability distribution and the finite size scaling of the lowest gaps and also the lowest energy level are derived using extreme statistics arguments (see also [32,33 and our shorter account of parts of this work 24]). These gaps have a logarithmic dependence on the number of local minima.

In order to derive the probability distribution of the first jump field $P\left(h_{1}\right)$, let us first calculate the probability $P_{0}$, that with a certain test field $h^{\prime}<h_{1}$, the interface has not changed yet, i.e.

$$
P_{0}\left(h_{1}>h^{\prime}\right)=1-P_{0}\left(h_{1} \leq h^{\prime}\right)=1-\int_{0}^{h^{\prime}} P(h) d h .
$$

By differentiating $P_{0}\left(h_{1}>h^{\prime}\right)$, one gets the probability distribution of the first jump field, since

$$
P\left(h_{1}\right)=\left.\frac{\partial}{\partial h} P_{0}\left(h_{1} \leq h^{\prime}\right)\right|_{h^{\prime}=h_{1}} .
$$

We assume all the minima to be non-correlated and well separated from each other, see Fig. 5(a). There we have a global energy minimum $E_{0}$ at $z_{0}$ (note, that here the energy values as well as the field contributions are normalized by $L^{D}$, constant with a fixed system size), and energy gaps $\Delta E_{1}=E_{1}-E_{0}$ and $\Delta E_{1^{*}}=E_{1^{*}}-E_{0}>\Delta E_{1}$ with energies $E_{1}$ at $z_{1}$ and $E_{1^{*}}$ at $z_{1^{*}}$, respectively. Then we apply the field $h$, i.e., we tilt the energy landscape, see Fig. (b). Due to the statistical tilt symmetry the external field $h$ is assumed not to change the shape of the random landscape. At the lowest tilt to move the interface, i.e., at field $h_{1}$, it moves to $z_{1^{*}}$, since with the field $h_{1} E_{0}\left(h_{1}\right)=$ $E_{0}+h_{1} z_{0}=E_{1^{*}}\left(h_{1}\right)=E_{1^{*}}+h_{1} z_{1^{*}}<E_{1}\left(h_{1}\right)=E_{1}+h_{1} z_{1}$. However, sometimes $E_{1}$ and $E_{1 *}$ coincide, so that the interface jumps to the true second lowest minimum. Now we index the positions of the $N_{z}$ minima as $z_{i}$ (need not to be in order), so that $h\left(z_{0}-z_{i}\right)=h \Delta z_{i}$, and we have

$$
\begin{aligned}
& P_{0}\left(h_{1}>h^{\prime}\right)=\prod_{i=1}^{N_{z}} \operatorname{prob}_{i}\left(\Delta E_{i}>h \Delta z_{i}\right) \\
& =\prod_{i=1}^{N_{z}}\left\{1-\int_{0}^{\Delta z_{i} h} \hat{P}_{i}\left(\Delta E_{i}\right) d\left(\Delta E_{i}\right)\right\} .
\end{aligned}
$$

By assuming all the gap energies from the same probability distribution $\hat{P}(\Delta E)$, setting $\Delta z_{k} \hat{=} \frac{k}{N_{z}}$ and taking the continuum-limit we get for the probability distribution of the first jump field using Eqs. (10), (11) and (12),

$$
\begin{array}{r}
P\left(h_{1}\right)=\exp \left[-\int_{1}^{N_{z}} \int_{0}^{k h_{1} / N_{z}} \hat{P}(\Delta E) d(\Delta E) d k\right] \times \\
\int_{1}^{N_{z}} \frac{\hat{P}\left(k h_{1} / N_{z}\right)}{1-\int_{0}^{k h_{1} / N_{z}} \hat{P}(\Delta E) d(\Delta E)} d k(13)
\end{array}
$$

There are two approaches to go on from the distribution of Eq. (13): one may either compare its prediction with numerics with a trial distribution for $\hat{P}$, or alternatively proceed by a complete Ansatz for the valley energies, from which the gap naturally follows. We now attempt the latter, and comment on the first one together with the numerics.

In order to calculate the probability distribution for the gap energies $\hat{P}(\Delta E)$, we use a probability distribution for the energy minima suitable for directed polymers, which has the advantage of decaying faster than any power-law, $\mathcal{P}(E) \sim \exp \left(-B E^{\eta}\right), \eta>0$. For directed polymers with the "single valley" boundary condition case, i.e., one end of the manifold fixed and thus no room for other minima, it is known mostly numerically that the bulk of the distribution of the energy of a directed polymer $\mathcal{P}(E)$ is Gaussian (the exponent $\eta=2$ in the exponential), except for the tails, $\left(E<E_{\min }\right.$ and $\left.E>E_{\max }\right)$ in which different cut-offs, $\left(\eta_{-}=1.6\right.$ and $\left.\eta_{+}=2.4\right)$ take over [34. 印. For the other manifolds that one might be interested in the actual distributions have not generally been studied. In the following we usually approximate the distribution with Gaussian distribution (this is valid for $1 / N_{z} \geq E_{\text {min }}$ and $\left.<E_{\max }\right)$. The validity of the approximation is discussed in Section 5.2, when the analytical arguments are compared with the numerics.

The distribution $(\eta=2$ for Gaussian) for $\mathcal{P}(E)$ is written in the form:

$$
\mathcal{P}(E)=k \exp \left\{-\left(\frac{|E-\langle E\rangle|}{\Delta E}\right)^{\eta}\right\}
$$

where $\langle E\rangle \sim L^{D}$ is the average energy of the manifold and $k \sim(\Delta E)^{-1} \sim L^{-\theta}$ normalizes the distribution. The extreme statistics argument goes so that in a system with 
E. T. Seppälä and M. J. Alava: Energy landscapes, lowest gaps, and susceptibility of elastic manifolds at $T=0$

$N_{z} \sim L_{z} / L^{\zeta}$ minima the probability for the lowest energy to be $E$ is

$$
L_{N_{z}}(E)=N_{z} \mathcal{P}(E)\left\{1-C_{1}(E)\right\}^{N_{z}-1},
$$

where $C_{1}(E)=\int_{-\infty}^{E} \mathcal{P}(e) d e$ is called the error-function when $\eta=2$. For Gaussian $\mathcal{P}(E)$, likewise for general $\eta>0$, $L_{N_{z}}(E)$ is known to be Gumbel distributed, $\exp (u-\exp u)$ 27, 28, 35. The gap $\Delta E_{1}$ follows similarly as $L_{N_{z}}(E)$. Its distribution, $G_{N_{z}}\left(\Delta E_{1}, E\right)$ is given by

$$
\begin{array}{r}
G_{N_{z}}\left(\Delta E_{1}, E\right)=\frac{N_{z}\left(N_{z}-1\right)}{2} \mathcal{P}(E) \mathcal{P}\left(E+\Delta E_{1}\right) \\
\left\{1-C_{1}\left(E+\Delta E_{1}\right)\right\}^{N_{z}-2}
\end{array}
$$

$G_{N_{z}}\left(\Delta E_{1}, E\right)$ is the probability that if the lowest energy manifold has an energy $E$, then the gap to the next lowest energy level is $\Delta E_{1} . G_{N_{z}}\left(\Delta E_{1}, E\right)$ is actually a generalization of Gumbel distribution. Integrating Eq. (16) over all energies gives the probability distribution for the $\Delta E_{1}$

$$
\hat{P}\left(\Delta E_{1}\right)=\int_{-\infty}^{\infty} G_{N_{z}}\left(\Delta E_{1}, E\right) d E
$$

This becomes for Gaussian $(\eta=2)$ and $\Delta E_{1} \ll\langle E\rangle$

$$
\hat{P}\left(\Delta E_{1}\right)=\int_{-\infty}^{\infty} \frac{N_{z}\left(N_{z}-1\right)}{2} k^{2} \exp \left\{\frac{-(E-\langle E\rangle)^{2}-2 \Delta E_{1}(E-\langle E\rangle)}{\Delta E^{2}}\right\}\left\{k \operatorname{erfc}\left(\frac{E+\Delta E_{1}-\langle E\rangle}{\Delta E}\right)\right\}^{N_{z}-2} d E
$$

where erf denotes the error-function and erfc $=1-$ erf. Neglecting all $\mathcal{O}\left[\left\{k \operatorname{erfc}\left(\frac{E+\Delta E_{1}-\langle E\rangle}{\Delta E}\right)\right\}^{2}\right]$ and higher order terms and using a Taylor expansion around $\Delta E_{1}=0$, i.e., Maclaurin-series, gives

$$
\begin{gathered}
\hat{P}\left(\Delta E_{1}\right)=\int_{-\infty}^{\infty} \frac{N_{z}\left(N_{z}-1\right)}{2} k^{2} \exp \left\{\frac{-(E-\langle E\rangle)^{2}-2 \Delta E_{1}(E-\langle E\rangle)}{\Delta E^{2}}\right\} \\
{\left[1-\left(N_{z}-2\right) k \operatorname{erf}\left(\frac{E-\langle E\rangle}{\Delta E}\right)+\left(N_{z}-2\right) \Delta E_{1} k \exp \left\{\frac{-(E-\langle E\rangle)^{2}}{\Delta E^{2}}\right\}\right] d E .}
\end{gathered}
$$


The first two terms within the second parenthesis dominate for $\Delta E_{1}$ small, and thus we see that to first order of $\hat{P}\left(\Delta E_{1}\right) \sim$ const (compare with the numerics presented below). In particular, one should notice that the probability distribution does not vanish for $\Delta E_{1} \simeq 0$.

This approach is very similar to the extreme statistics calculation of the one-dimensional version of the random energy model by Bouchaud and Mézard [27] (see Section 2). They derived the probability distribution for the location that gives minimum energy for the system, with the difference to our case that Hamiltonian reads $\frac{\gamma}{2} x^{2}+E(x)$. The result becomes such that the distribution for the position is approximately Gaussian, too. The one-dimensional system is close to our example except for the functional form of the external potential which is quadratic instead of a linear one. There is however one essential difference in that in their analysis the "field value" is fixed, whereas in our case we are interested in what happens in any particular sample as the field is varied. Nevertheless such a calculation could be compared to the distribution of the interface locations at a fixed field $h$.

More important than the actual distributions is that the finite size scaling of the gap energies may be computed. Let us start by calculating the typical lowest energy level. The average of it is given by

$$
\left\langle E_{0}\right\rangle=\int_{-\infty}^{\infty} E L_{N_{z}}(E) d E
$$

and the typical value of the lowest energy is estimated from

$$
N_{z} \frac{1}{k} \mathcal{P}\left(\left\langle E_{0}\right\rangle\right) \approx 1
$$

Note, that in approximating the integral, Eq. (20), with the aid of the distribution, Eq. (21) (in the limit $C_{1}(E)$ in Eq. (15) is small) the normalization $1 / k$ should be taken into account. Eq. (21) gives

$$
\left\langle E_{0}\right\rangle \sim\langle E\rangle-\Delta E\left[\ln \left(N_{z}\right)\right]^{1 / \eta},
$$

where $\Delta E \sim L^{\theta}$ and for Gaussian $\eta=2$.

To estimate the typical value of the gap, we make a similar approximation as in Eq. (21) for $\left\langle E_{0}\right\rangle$,

$$
\frac{N_{z}\left(N_{z}-1\right)}{2 k^{2}} \mathcal{P}\left(\left\langle E_{0}\right\rangle\right) \mathcal{P}\left(\left\langle E_{0}\right\rangle+\left\langle\Delta E_{1}\right\rangle\right) \approx 1,
$$

which with (22) and the fact that $\left|\left\langle\Delta E_{1}\right\rangle\right| \ll\left|\left\langle E_{0}\right\rangle\right|$ yields,

$$
\left\langle\Delta E_{1}\right\rangle \approx \frac{\Delta E^{\eta}}{\eta\left(\langle E\rangle-\left\langle E_{0}\right\rangle\right)^{\eta-1}} \approx \frac{\Delta E}{\eta\left[\ln \left(N_{z}\right)\right]^{(\eta-1) / \eta}} .
$$

We thus find that the gap scales as $\Delta E_{1} \sim \Delta E\left[\ln \left(N_{z}\right)\right]^{-1 / 2}$ $L^{\theta}\left[\ln \left(L_{z} L^{-\zeta}\right)\right]^{-1 / 2}$, when $\eta=2$ and assuming as in the previous section, that $N_{z} \sim L_{z} / L^{\zeta}$. If the interfaces are flat, i.e., $\zeta=0$, which is true for $\{100\}$ RB interfaces below the cross-over roughening scale $L_{c}$ 36, 37, 38 , 39 if randomness is weak, the same arguments should hold. However, then the energy distribution is pure Gaussian, $\eta=2$, and $\theta=D / 2$ due to Poissonian statistics.

\section{Susceptibility of manifolds}

\subsection{Numerical method}

For the numerical calculations the RB Hamiltonian (1) with $J_{i j}>0, H=0$, has been transformed to a random flow graph. The graph is formed by the Ising lattice and two extra sites: the source and the sink; and the coupling constants $2 J_{i j} \equiv c_{i j}$ between the spins correspond to flow capacities $c_{i j} \equiv c_{j i}$ from a site $S_{i}$ to its neighboring one $S_{j}$ [40]. The graph-theoretical optimization algorithm, a maximum-flow minimum-cut algorithm, enables us to find the bottleneck, which restricts the amount of the flow that can get from the source to the sink given the capacities in such a random graph. This bottleneck, a path $P$ which divides the system into two parts (sites connected to the sink and sites connected to the source) is the minimum cut of the graph and the sum of the capacities belonging to the cut $\sum_{P} c_{i j}$ equals the maximum flow, the smallest of all cut-paths in the system. The source is connected to the sites in the Ising lattice which are forced to be up and the sink is connected to the sites which are forced to be down. The value of the maximum flow is the total minimum energy of the domain wall, equivalent to the minimum cut. The maximum flow algorithms can be proven to give the exact minimum cut for all the random graphs, in which the capacities are positive semidefinite and with a single source and sink 411. The best known maximum flow method is by Ford and Fulkerson and called the augmenting path method 42. We have used a more sophisticated method called push-and-relabel by Goldberg and Tarjan [43], which we have optimized for our purposes. It scales almost linearly, $\mathcal{O}\left(n^{1.2}\right)$, with the number of spins and gives the ground state DW in about minute for a million of spins in a workstation. Notice that one could use for DP's the usual transfer matrix method as well, but the max-flow implementation is convenient in the case of systematic perturbations to each sample.

In this study we have done simulations for $(1+1)$ and $(2+1)$ dimensional manifolds. The system sizes extend to $L \times L_{z}=1000^{2}$ and $L^{2} \times L_{z}=400^{2} \times 50$. The number of realizations $N$ ranges from 200 to $2 \times 10^{4}$. The random bonds are such that in $2 \mathrm{D} J_{i j, z} \in[0,1]$ uniform distribution and $J_{i j, x}=0.5$ unless otherwise mentioned. In three dimensions we have either $J_{i j} \in[0,1]$ uniform distribution in all $x, y$, and $z$ directions or dilution type disorder, $P\left(J_{i j}\right)=p \delta\left(J_{i j}-1\right)+(1-p) \delta\left(J_{i j}\right)$, i.e., a bond has a value of unity or zero with the probability $p$. We have used $p=0.5$ and $p=0.95$. The linear field contribution is applied in the $z$-directional bonds as $J_{\perp}(z)=J_{\text {random }}+h z$. When the jump field values with $\sim$ the corresponding jump distances are searched, the precision is as small as $\Delta h=10^{-5}$ in order to be sure that no smaller changes would occur between the jumps, which could be the case if the precision was much larger. Periodic boundary conditions are used in $x$ and $y$ directions.

When studying the susceptibility, we have controlled the number of the minima in the systems to avoid fluctuations in $N_{z}$ (and the grand-canonical ensemble). To fix 
the number of the minima $\left\langle N_{z}\right\rangle \sim L_{z} / L^{\zeta}$ in a certain system size, we have set the initial position of the interface $\bar{z}_{0}$ in a fixed size window at height $\bar{z}_{0} / L_{z} \simeq$ const. If the ground state interface is originally outside the window, large enough only for a single valley, it is discarded so that the statistics quoted are based on the successful attempts. If the window is well-separated in space from the $z$-directional boundaries it is obvious that this sampling has no effect on the statistical properties as the average energy. After an original ground state is found, with an energy $E_{0}$, the external field $h$ is applied by increasing the couplings by constant steps until the first jump is observed 44]. We have also calculated the energy gap between the global minimum and the next lowest minimum. In that case the initial position of the interface $\bar{z}_{0}$ is also set to be in a fixed size window at height $\bar{z}_{0} / L_{z} \simeq$ const by discarding all the samples with the global minimum outside the window. After that the lattice is reduced so that bonds in and above the window are neglected and the new ground state, its energy $E_{1}$, and the corresponding gap energy $\Delta E_{1}$ are found. Although the discarding procedure is slow we have at least $N=500$ realizations up to system sizes $L=300, L_{z}=500$.

\subsection{Results}

In order to compare the analytical arguments presented in Section 1 and in the end to compute the total susceptibility of the manifolds we will first study numerically in $(1+1)$ dimensions the finite size scaling of the average lowest energy level. Then the shape of the energy gap distribution as well as the finite size scaling of the average gap energies are considered. After that the numerical results of the first jump field distribution are presented together with the finite size scaling of the average first jump field. The shape of the first jump field distribution and the finite size scaling of its average are shortly reported for $(2+1)$ dimensional interfaces, too. The susceptibility is derived from the distribution and the finite size scaling of the first jump field. In the end of the section the jump distance distributions are considered.

In order to see the logarithmic correction of the lowest energy level $\left\langle E_{0}\right\rangle$, Eq. (22), when the height of a system, and thus the number of the minima, are increased, we have plotted in Fig. 6 for three different lengths of the directed polymers $L=100,200$, and 300

$$
\frac{\langle E\rangle-\left\langle E_{0}\right\rangle}{L^{\theta}} \sim\left[\ln \left(N_{z}\right)\right]^{1 / 2},
$$

where $N_{z} \sim L_{z} / L^{\zeta}$, and $\langle E\rangle \simeq 0.365 L+1$. The prefactor, 0.365 , of the average energy of a polymer with only a single valley in a system, $\langle E\rangle$, we have estimated by calculating systems of sizes $L_{z} \times L$, where $L_{z}=6.5 \times w, w$ is the average roughness of a polymer, up to system sizes $50 \times 1000$ with 2000 realizations. One should note, that the prefactor is highly sensitive to the disorder type and boundary conditions, c.f. Ref. 45, and the constant factor (unity) also to the estimate of the size of the single valley. We have used it as a free parameter. Although for the following results the relevant part of the tail of $\langle E\rangle$ should not be a pure Gaussian (at least if we have enough many minima $N_{z}$, so that the bulk of the distribution is avoided), we have used for $1 / \eta=1 / 2$ instead of $1 / \eta=1 / 1.6=0.625$ in the fit of the logarithmic correction. In practice one can not tell these two choices apart in the range of the system sizes used.

The probability distributions for the energy gaps of directed polymers in system sizes $L^{2}=100^{2}$ and $200^{2}$ are shown in Fig. I(a). The distribution has a finite value at $\Delta E_{1}=0$ and the tail has approximately a stretched exponential behavior, $\exp \left(-a x^{b}\right)$, with an exponent $b \simeq 1.3$. In the figure there is plotted as a comparison an exponential $\exp (-x)$ line, from which the deviation of the probability distribution is more clearly seen in the inset, where the distribution is in a natural-log scale. The finite value at $\Delta E_{1}=0$ is consistent with the weak replica symmetry breaking picture.

To derive the scaling function for the $\Delta E_{1}$ it is expected that in systems with height $L_{z}$ small enough to restrict the number of minima $\Delta E_{1}$ mainly depends on the height of the system $L_{z}$. On the other hand, when $L_{z}$ is large enough, there are enough valleys of which to choose the two minima, and one has $\Delta E_{1} \sim \Delta E \sim L^{\theta}$, hence

$$
\left\langle\Delta E_{1}\left(L, L_{z}\right)\right\rangle \sim \begin{cases}\tilde{f}\left(L_{z}\right), & L_{z} \ll L \\ L^{\theta}, & L_{z} \gg L\end{cases}
$$

when the smaller parameter being varied. Since it is assumed, that $L_{z} \sim L^{\zeta}$, a natural scaling form based on these limiting behaviors is,

$$
\left\langle\Delta E_{1}\left(L, L_{z}\right)\right\rangle \sim L^{\theta} f\left(\frac{L_{z}}{L^{\zeta}}\right) .
$$

The argument $y=L_{z} / L^{\zeta}$ for the scaling function $f(y)$ is just a function of the number of the minima, i.e., $L_{z} / L^{\zeta} \sim$ $N_{z}$, and the scaling function has the form from Eq. (24), when $\eta=2$,

$$
f(y) \sim[\ln y]^{-1 / 2}
$$

In Fig. $7(\mathrm{~b})$ we have plotted the scaling function (28) by collapsing $\left\langle\Delta E_{1}\left(L, L_{z}\right)\right\rangle / L^{\theta}$ versus $L_{z} / L^{\zeta}$ for various $L$ and $L_{z}$, and find a nice agreement confirming the scaling behavior Eqs. (27), (28) as well as the analytic form Eq. (24) again assuming a Gaussian distribution $(\eta=2)$.

Next we explore the first jump fields. Consider the relation of the gap distribution and that of the jump fields given by Eq. (13). If we approximate $\hat{P}\left(\Delta E_{1}\right)$, Eq. (19), with a uniform distribution, we get for the probability distribution of the first jump field

$$
P\left(h_{1}\right)=\exp \left[-\frac{N_{z} h_{1}}{2}\right] \frac{N_{z}}{h_{1}} \ln \left[\frac{1-\frac{h_{1}}{N_{z}}}{1-h_{1}}\right] \sim \exp \left(-h_{1}\right) .
$$

The form of $P\left(h_{1}\right)$ implies that it has a finite value at $h_{1}=0$, which is again consistent with the weakly broken replica symmetry picture [25] and also with the discussion in Section 4 . We have also tried exponential and 
power-law type of probability distributions for $\hat{P}\left(\Delta E_{1}\right)$ in Eq. (13), but all trials with negative exponents vanishes too fast with $h_{1}$ compared to the numerical data, and all positive exponents have behaviors with $P\left(h_{1} \rightarrow 0\right) \rightarrow 0$ and diverge for larger $h_{1}$.

In Fig. 8(a) we have plotted the probability distribution of the first jump field for the system sizes $L^{2}=100^{2}$ and $200^{2}$. The probability distribution of the first jump field is similar to the probability distribution of the gap energies. The analytic formula, Eq. (29), is drawn as a line in the figure. One clearly sees that the line is a pure exponential, $\exp (-x)$, and the deviation of the numerical data from the exponential is similar to Fig. 7 (a) of the energy gap distributions. Hence the shape of the numerical first jump field distribution is approximately a stretched exponential, $\exp \left(-a x^{b}\right)$, with an exponent $b \simeq 1.3$. Based on Figs. 7(a) and 8(a) one sees, that the probability distributions of the energy gaps and first jump fields are the same. Thus with the correct $\hat{P}(\Delta E)$ one gets from Eq. (13) the same $P\left(h_{1}\right)=\hat{P}(\Delta E)$. This distribution may be for small $h_{1}$ flat, but obviously starts to vanish for larger $h_{1}$ since $P$ decays faster than exponentially. Another reason can be the fact that by discarding samples with the GS outside of a predefined window we just constrain the number of valleys in the sample so that the expectation value is the same in each one. $N_{z}$ can however fluctuate from sample to sample. The most important consequence is in any case that $\hat{P}\left(\Delta E_{1}=0\right)=P\left(h_{1}=0\right) \neq 0$. The finite size scaling and the normalization of the probability distribution of $\Delta E_{1}$ give $\hat{P}\left(\Delta E_{1}=0\right) \sim L^{-\theta}\left[\ln \left(L_{z} L^{-\zeta}\right)\right]^{1 / \eta}$.

In order to find the scaling relation for the first jump field $h_{1}$, we make the Ansatz $\left\langle\Delta E_{1}\right\rangle=\left\langle h_{1}\right\rangle L L_{z}$, since the field contributes to the manifold energy proportional to $L^{D}(D=1)$ and $L_{z} \sim\left\langle\Delta z_{1}\right\rangle$ is the difference in the field contributions $h z$ to the energy at finite $h$ at different average valley heights $z_{0}, z_{1}$. Hence

$$
\left\langle h_{1}\left(L, L_{z}\right)\right\rangle L L_{z} \sim L^{\theta} f\left(\frac{L_{z}}{L^{\zeta}}\right),
$$

where the scaling function $f(y)$ for the number of the minima $N_{z} \sim L_{z} / L^{\zeta} \sim y$ has the same scaling function Eq. (28). Fig. 8(b) shows the scaling function (28) with a collapse of $\left\langle h_{1}\left(L, L_{z}\right)\right\rangle L^{1-\theta} L_{z}$ versus $L_{z} / L^{\zeta}$ for various $L$ and $L_{z}$ with a good agreement, again.

Next we move over to the $(2+1)$ dimensional manifolds. The inset of Fig. 9 shows the tail of the distribution for the system size $L^{3}=50^{3}$ with dilution type of disorder and bond occupation probability $p=0.5$. The first nonoverlapping jumps are included in the distribution, since due to the anomaly of the dilution disorder (lots of small scale degeneracy), there are typically two adjustments in the interface before the large jump. As a comparison, we plot again the $\exp (-x)$ line, too. One sees that the deviation of the tail of the distribution from the exponential behavior is similar to the $(1+1)$ dimensional case. The finite scaling of the first jump field in Fig. 9 is shown for interfaces of size $L_{x} \times L_{y}=50^{2}$ and systems of height $L_{z}=30-90$. We have fitted for a constant $L$ the formula
(30), i.e., $\left\langle h_{1}\left(L_{z}\right)\right\rangle \sim L_{z}^{-1}\left[\ln \left(L_{z}\right)\right]^{-1 / 2}$ and it works within error bars.

Generalizing the numerical results of $(1+1)$ and $(2+$ 1) dimensional calculations and the analytic arguments from the previous section to arbitrary dimensions gives the behavior of $\left\langle h_{1}\left(L, L_{z}\right)\right\rangle \sim L^{\theta-D} L_{z}^{-1}\left[\ln \left(L_{z} / L^{\zeta}\right)\right]^{-1 / 2}$. Since the probability distribution has a finite value at $P\left(h_{1}=0\right)$ and $\left\langle h_{1}\left(L, L_{z}\right)\right\rangle$ vanishes with increasing system size, one obtains from the normalization factor at $P\left(h_{1}=0\right)$ for the scaling of the susceptibility, Eq. (9)

$$
\chi \sim L^{D-\theta} L_{z}\left[\ln \left(L_{z} / L^{\zeta}\right)\right]^{1 / 2}
$$

and in the isotropic limit, $L \propto L_{z}$, the total susceptibility $\chi_{\text {tot }}=L^{d} \chi$ becomes

$$
\chi_{t o t} \sim L^{2 D+1-\theta}[(1-\zeta) \ln (L)]^{1 / 2}
$$

Thus the extreme statistics of energy landscapes shows up in the susceptibility of random manifolds or domain walls in a form that Eq. (32) has a logarithmic multiplier and the scaling behavior of the first jump field is due to the scaling of the energy minima differences. This is in contrast to Shapir's result [29 and the logarithmic contribution is also missing from our earlier paper [23] where the valley energy scale was taken to follow the standard $L^{\theta}$ assumption. Therefore one can conclude that the effect of extreme statistics is important in this problem: since we look at the finer details of the landscape "typical" differences are not sufficient. Notice that for two dimensional random field Ising magnets $\zeta \simeq 1$ at large scales [18] and the susceptibility does not diverge 46]: the premise that $N_{z}>1$ is broken in that case. If the condition $N_{z}>1$ is violated the extreme statistics correction disappears. For flat interfaces so that the effective roughness exponent is zero $\left(\zeta_{\text {eff }}=0\right)$, e.g. $\{100\}$ oriented RB interfaces with weak disorder and small system sizes $L<L_{c}$, when $\zeta=0$ 36, 37, 38, 39, $\theta=D / 2$ and Eq. (32) becomes $\chi_{\text {tot }} \sim L^{2 D+1-D / 2}[\ln (L)]^{1 / 2} \sim L^{4}[\ln (L)]^{1 / 2}$, when $D=2$.

Finally we report the jump distance distributions of the first jumps. Fig. 10(a) shows $P\left(\Delta z_{1} / \bar{z}_{0}\right)$ of the first jump with the field in $(1+1)$ dimensions. The distribution is a superposition of two behaviors: the interface jumps to the lowest minimum as such, and the external field favors the minima close to the wall (remember the differences of $z_{1}$ and $z_{1^{*}}$ in Fig. 5). Since the non-overlapping cases are excluded and the wall has a repulsive effect, there are cut-offs in the both ends of the distributions. The shape of the distribution does not change with the system size, which is consistent with the assumptions in Eqs. (9) and (30), $\left\langle\Delta z_{1}\right\rangle \sim L_{z}$. Fig. 10(b) shows the same distribution of $P\left(\Delta z_{1} / \bar{z}_{0}\right)$ with a field for $(2+1)$ dimensional manifolds and it is clear that the shape of the distribution does not depend on the dimension. In Fig. 10(c) we have plotted $P\left(\Delta z_{1} / \bar{z}_{0}\right)$ without a field, i.e., the distance of the true lowest energy minima in $(1+1)$ dimensions. Now there is no field, and thus the shape of the distribution is just a uniform one, again consistent with the predictions made in Section 4 in Eq. (12). 


\section{Scaling of the first jumps: field and distance}

Following similar arguments as Eq. (8) in Section 3 for an excitation, a mean field result for the finite size scaling of the first jump field is next derived. Let us have an interface at an arbitrary height $z_{0}$ with an energy $E_{0}$ and an isotropic system $L \propto L_{z}$. The energy gap between the two lowest energy minima, Eq. (24), scales as $\Delta E_{1} \sim L^{\theta}[(1-\zeta) \ln (L)]^{-1 / 2}$, when the $z_{0} \simeq L_{z}$. However, when the interface is at an arbitrary height the number of the available minima depends on the original position of the interface, and we use only the dominating algebraic behavior so that $\Delta E_{1} \sim L^{\theta}$.

On the other hand the energy difference of elastic manifolds at different heights due to the field contribution is $\Delta E \simeq h \Delta z L^{D}$. Assuming that $\langle\Delta z\rangle \sim L$ as in the earlier arguments, the field contribution becomes $\Delta E \sim h L^{d}$. We expect that the first jump happens, when the gap equals the field energy, and thus the first jump field has a scaling

$$
\left\langle h_{1}\right\rangle \sim L^{\alpha}=L^{\theta-d} .
$$

In Figs. 11(a) and 11(b) we have plotted the average first jump field $\left\langle h_{1}\right\rangle$ in isotropic systems for $(1+1)$ and $(2+1)$ dimensional manifolds, respectively. The data is taken from non-overlapping jumps, to minimize finite size effects since they are smaller than with the overlapping jumps included. However, the fraction of overlapping jumps is small as seen in Fig. 4 for $(1+1)$ dimensional case. The data in Figs. 11 confirms within error bars the expected behavior of $\left\langle h_{1}\right\rangle \sim L^{-5 / 3}$ and $\left\langle h_{1}\right\rangle \sim L^{-2.18}$ for $(1+1)$ and $(2+1)$ dimensional manifolds, respectively. The logarithmic term $\left[\ln \left(N_{z}\right)\right]^{-1 / 2}$ might contribute a downward trend since it decreases with the number of the available minima $N_{z}$, here disorder averaged due to the arbitrary GS location. This is however not noticeable in the data: the finite size effects in the calculated data are towards smaller absolute value of the exponent. In the insets of Figs. 11(a) and (b) the linear scaling of jump sizes $\left\langle\Delta z_{1}\right\rangle \sim L$ is confirmed, which was assumed in Eqs. (9) and $(30)$.

The evolution of the jump size distribution for succeeding jumps is demonstrated in Fig. 12. As a comparison for Fig. 10 where only the non-overlapping jumps were considered, here we have shown the overlapping ones, too. It is seen as a peak near $\Delta z_{n} / \bar{z}_{n-1}=0$. The first jumps have clearly the most weight in the large jump end of the distribution, but for the following jumps the whole distribution shifts towards $\Delta z_{n} / \bar{z}_{n-1}=0$. After a small number of jumps the interface is already in the minimum closest to the wall and then the random-bulk wetting behavior takes over. In order to estimate the number of the jumps the manifold does before binding to the wall, it is assumed that after a jump the system looks statistically the same as before it. From Fig. 10(a) we infer that the probability distribution for the jump size has a form $P(\Delta z) \sim \Delta z^{2}$ (note that we did not attempt to compute this from the analytical valley arguments) and that is then taken to be the same for all (large) jumps $\Delta z_{n}$. To calculate the average jump size of jumps which do not jump to the closest valley to the wall we have

$$
\begin{array}{r}
\left\langle\Delta z_{n}\right\rangle=\int_{0}^{z_{n-1}-A_{1} w} B_{n-1} \Delta z(\Delta z)^{2} d(\Delta z) \\
=\frac{3\left(z_{n-1}-A_{1} w\right)^{4}}{4 z_{n-1}^{3}}
\end{array}
$$

where the upper bound of the integral is to neglect the cases that jump to the closest valley to the wall, $A_{1}$ is the prefactor to multiply the roughness value to get the valley width, and $B_{n-1}=3 / z_{n-1}^{3}$ normalizes the probability distribution. Using $\left\langle z_{n}\right\rangle=\left\langle z_{n-1}\right\rangle-\left\langle\Delta z_{n}\right\rangle$ we get the next position of the interface. In order to calculate the probability of an interface to jump with the first jump to the closest valley to the wall, $p_{1}$, we use the same probability distribution as in Eq. (34),

$$
p_{1}=\int_{z_{0}-A_{1} w}^{z_{0}} B_{0}(\Delta z)^{2} d(\Delta z)=1-\left[1-\frac{A_{1} w}{z_{0}}\right]^{3}=\tilde{p}_{1},
$$

and similarly for the probability of an interface to jump with the second jump to the closest valley to the wall

$$
\begin{aligned}
p_{2}=\left(1-\tilde{p}_{1}\right) \tilde{p}_{2} & =\left(1-\tilde{p}_{1}\right) \int_{z_{1}-A_{1} w}^{z_{1}} B_{1}(\Delta z)^{2} d(\Delta z) \\
& =\left[1-\frac{A_{1} w}{z_{0}}\right]^{3}\left\{1-\left[1-\frac{A_{1} w}{z_{1}}\right]^{3}\right\}
\end{aligned}
$$

Due to the hierarchy we finally get for the $n^{\text {th }}$ jump

$$
p_{n}=\tilde{p}_{n} \prod_{k=1}^{n-1}\left(1-\tilde{p}_{k}\right)=\left[1-\left(1-q_{n}\right)^{3}\right] \prod_{k=1}^{n-1}\left(1-q_{k}\right)^{3}
$$

where

$$
q_{n}=\frac{A_{1} w}{z_{n-1}} .
$$

To get an estimate for the number of jumps, we write

$$
\langle\mathcal{N}\rangle=\sum_{n=1}^{\infty} n p_{n} .
$$

This can be evaluated, but only approximately since among others the estimate of Eq. (37) breaks down beyond $n$ small. Using $A_{1} w \simeq A L^{\zeta}=50, L=1000$, which is the case with $A_{1} \simeq 6.5$, (see the numerics in Section 5.2), and taking $\bar{z}_{0}=1000$, we get $\langle\mathcal{N}\rangle \simeq 3$. Eq. (34) gives for the first jump size, with the above numerical values, $\left\langle\Delta z_{1}\right\rangle \simeq 0.6 \bar{z}_{0}$, which is not far from the behavior in the inset of Fig. 11(a), where $\left\langle\Delta z_{1}\right\rangle \sim 0.4 L_{z} \sim 0.8 \bar{z}_{0}$, since $\left\langle\bar{z}_{0}\right\rangle \sim L_{z} / 2$.

\section{Application of a non-zero field: random-bulk wetting}

In order to see the wetting behavior one just studies the effect of a large external field on the average interface to 
wall distance. Notice that the low-field physics discussed extensively above can be considered as the eventual outcome in any sample with $L_{z}>L^{\zeta}$ so that more than one valley is available. This means that when the field is decreased from a large field value, the interface finally jumps into the bulk. Fig. 13(a) we show the average mean height $\langle\bar{z}(h)\rangle$ versus the field $h$ for $(1+1)$ and $(2+1)$ dimensional manifolds. The system sizes are $L_{z} \times L=100 \times 3000$ and $L_{z} \times L^{2}=50 \times 300^{2}$. To maximize the prefactor, $A_{2}$, in the scaling of roughness, $w \sim A_{2} L^{\zeta}$, and hence the width of the minimum energy valley, the disorder has been chosen to be strong, i.e., dilution type of disorder with small $p$, but above the bond-percolation threshold. However, there are still some deviations in the form of greater exponents than the expected from Eq. (7), which gives the values $\psi=1 / 2$ and $\psi \simeq 0.26$ in $(1+1)$ and $(2+1)$ dimensions from $\zeta_{(1+1)}=2 / 3$ and $\zeta_{(2+1)}=0.41 \pm 0.01$, respectively. We found that the trend is nevertheless clear, with greater $L$ and fixed $L_{z}$ the exponents become closer to the expected one. The effective exponent $\psi_{\text {eff }}(L)$ can be used to extract the asymptotic, $L$-independent exponent in particular in $(2+1)$ dimensions since this case is hampered most by finite-size effects. $\psi_{\text {eff }}(L)$ as a function of $1 / L$ indicates that the asymptotic value is indeed $0.26 \pm 0.02$ and that the system sizes at which $\psi_{\text {eff }}(L)$ approaches that are of the order of $L=10^{4}$. That is, only with $10^{10}$ sites it becomes possible to reach the asymptotic regime. When one has $L \propto L_{z}$ calculating the average mean height $\langle\bar{z}(h)\rangle$ with a fixed field $h$ is nothing but averaging over the jumped and not jumped interfaces together with their location, see Fig. 2(a).

In Fig. 13(b) we have plotted as a comparison the average mean height $\langle\bar{z}(h)\rangle$ versus the field $h$ for $(2+1)$ dimensional manifolds with a weak disorder. In this case the weak means for system sizes used, that the roughness of the manifolds are not in the asymptotic roughness limit yet, $L<L_{c}$ 36, 37, 38, 39. The system sizes used are $L_{z} \times L^{2}=50 \times 80^{2}-400^{2}$ and the behavior is simple: either the interface stays in its original position or jumps directly to the wall. Taking into account the jumped and original interfaces as $\langle z(h)\rangle=\langle z[1-$ $\left.\left.P\left(\bar{z}_{0}, h\right)\right] \bar{z}_{0}+P\left(\bar{z}_{0}, h\right) \times 0\right\rangle=\left\langle\left[1-P\left(\bar{z}_{0}, h\right)\right] \bar{z}_{0}\right\rangle=\int_{0}^{L_{z}}[1-$ $\left.P\left\{\bar{z}_{0}(h)\right\}\right] \bar{z}_{0} d \bar{z}_{0}$, gives the behavior $\langle z(h)\rangle \sim h^{-1}$, i.e., the exponent $\psi \simeq 1$, if $P\left\{\bar{z}_{0}(h)\right\} \sim h^{-1}$. The larger manifolds jump faster to the wall, i.e., they feel the perturbation earlier, since Eq. (30) for flat interfaces $(\zeta=0)$ becomes $\left\langle h_{1}\left(L, L_{z}\right)\right\rangle \sim L^{\theta-D} L_{z}^{-1}\left[\ln \left(L_{z}\right)\right]^{-1 / 2}$. With fixed $L_{z}$ and $\theta=D / 2$ from Poissonian statistics, we get in $D=2\left\langle h_{1}(L)\right\rangle \sim L^{-1}$ consistent with the numerical data. This leads to the behavior of the wetting scaling, $\langle\bar{z}(h)\rangle \sim c(L) h^{-\psi}$ where $c(L) \simeq L^{-1}$ and $\psi=1$. The finite size scaling of the prefactor indicates that at large $L$-limit with fixed $L_{z}$ the flat interfaces are immediately at the wall, and thus the systems are non-wet. This implies that there is an interesting cross-over around $L_{c}$ between such a "dry" regime and the bulk wetting that takes over for still larger $L$. In $D>2$ this kind of behavior is relevant even in the thermodynamic limit, if the disorder is weak due to the presence of a bulk roughening transition for bond disorder.

\section{Discussion}

\subsection{Finite temperature behavior}

The movement of the elastic manifolds in random media at low temperatures, when an applied force is much below the depinning threshold $F_{c}$, is characterized as creep. The dynamics is controlled by thermally activated jumps over pinning energy barriers, which separate the metastable states. D. Fisher and Huse 19] showed that for a DP at finite temperature $T>0$ the fluctuations of the entropy $(\Delta S)^{2}$ and the internal energy $\left(\Delta E_{\text {int }}\right)^{2}$ scale linearly with the length of the polymer and cancel each other. Hence there are only the fluctuations of the free energy $(\Delta F)^{2}$, which scale with the zero temperature energy fluctuation exponent $2 \theta=2 / 3$. Since the free energy is the one which should be minimized at finite temperature, it is the one which defines statistically the shape of the energy landscape, although the energy valleys and minima need not to have exactly the same real space structure as at $T=0$. Thus the $\theta=1 / 3$ exponent should define the energy gaps also when $T>0$, expect in the cases there is a critical temperature $T_{c}$. Hence, our derivation of the susceptibility and also the first order character in the reorganization of valleys should be relevant also at $T>0$.

\subsection{First arrival times in nonlinear surface growth}

The $(1+n)$ dimensional directed polymers map, in the continuum limit, to the KPZ [3, 47] equation by associating the minimum energy of a DP-configuration with the minimum arrival time $t_{1} \equiv E_{0}$ of a KPZ-surface to height $H$. The connection is illustrated in Fig. 14. The minimal path of the DP with the end point $\mathbf{x}_{1}\left(t_{1}\right)$ equals the path by which the interface reaches $H$, at location $\mathbf{x}_{1}$ and at time $t_{1}$. Thus $t_{1}$ attains a logarithmic correction, from Eq. (22), of size $-H^{\beta}\left\{\ln \left(L / H^{1 / z}\right)\right\}^{1 / \eta}$, where $L$ is the linear size of the system, $\beta=\theta>0$ and $z=1 / \zeta$ are now the roughening exponent and dynamical exponent of the KPZ universality class and the values of $\theta=2 \zeta-1$ and $\zeta>1 / 2$ depend on $n$. Notice that if there is a upper critical dimension $n_{c}$ in the KPZ growth, then the logarithmic correction is not there anymore, and $\theta=0, \zeta=1 / 2$, i.e., a random walk ensues. Consider now the second smallest arrival time $t_{2}$. In the language of directed polymers, if the path $\mathbf{x}_{2}\left(t^{\prime}\right)$ corresponding to $t_{2}$ is independent of $\mathbf{x}_{1}\left(t^{\prime}\right)$ that corresponds to $t_{1}$ the time and the path are found inside a separate, independent valley. The difference $\Delta t=t_{2}-t_{1}$ then equals $\Delta E_{1}$, and likewise obeys extreme statistics, so that $\Delta t \sim H^{\beta}\left[\ln \left(L / H^{1 / z}\right)\right]^{-(\eta-1) / \eta}$. For growing surfaces this limit is the early stages of growth, in which the correlation length $\xi \ll L$, and therefore the arrival times, or directed polymer energies, are independent. On the other hand if we disturb the growing process such a way that it depends 
E. T. Seppälä and M. J. Alava: Energy landscapes, lowest gaps, and susceptibility of elastic manifolds at $T=0$

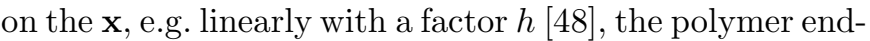
ing at $\mathbf{x}_{2}$ becomes faster if $h>h_{1}$, see right hand side of Fig. 14. Similarly now the factor $h_{1}$ has a scaling behavior from Eq. (30), $h_{1} \sim H^{\beta-1} L^{-1}\left[\ln \left(L / H^{1 / z}\right)\right]^{-(\eta-1) / \eta}$.

\section{Conclusions}

To conclude we have studied the $(1+1)$ and $(2+1)$ dimensional elastic manifolds at zero temperature, when an external field is applied. We have demonstrated that the response of manifolds shows a first order character ("jump") in the sense that the manifolds change their configuration in the large system size limit completely. This persists in a finite system over a small number of such jumps. The distance that the center-of-mass moves is extensive. The whole picture is based on a level-crossing between two lowenergy valleys in the energy landscape. Averaging over the total magnetization of such random magnets with a domain wall or over the positions of manifolds when an external field is applied becomes dependent on whether the DW or manifold has jumped or not. This leads to the problem of self-averaging in random systems. Here a disorder average smooths over the "coexistence" between systems that are not affected by a finite field $h$ and those that have responded.

In order to study the susceptibility of the DW in random media, one has to take into account the probability distribution of the sample-dependent field associated with the global change of the configuration, and take the limit of vanishing fields. This probability distribution has a finite density at $h=0$. The finite size scaling of the density is dependent on the finite size scaling of the number of the low lying nearly degenerate energy minima in the system. The scaling of the number of the energy minima leads to a logarithmic factor in the susceptibility, and can be accounted for by using extreme statistics. Such effects are difficult to study by usual field theoretical means, since one has to have access to the whole probability distribution and not only the few first moments thereof. Notice that the crucial difference to much previous work is the simple fact that we allow for multiple minima in the energy landscape, which is often most excluded by the boundary conditions applied to the problem. Although the derivations and the numerical calculations done here have concentrated on random bond type of randomness similar behavior should be seen in random field cases, too.

The discrete character in the movement of elastic manifolds with an external field results also in that the continuum theory for wetting in random systems works only in slab geometries, where there is room only for a single valley, or in the large external field limit, when the interface is close to the wall. On the other hand the flat interfaces are shown to jump directly to the wall, i.e., to be non-wet. It would be interesting to see if the dynamics of the manifolds at finite temperature reflects the first order character seen here at $T=0$. Through the connection of $(1+1)$ dimensional DW's to the directed polymers of the KPZ surface growth, we have shown that the logarithmic factor is also present in the statistics of growth times in nonlinear surface growth.

The authors would like to thank the Academy of Finland's Centre of Excellence Programme for financial support and the Center for Scientific Computing, Espoo, Finland for computing resources. Phil Duxbury and Simone Artz are acknowledged for many valuable discussions.

\section{References}

1. D. Fisher, Phys. Rev. Lett. 56 (1986), 1964.

2. T. Emig and T. Nattermann, Eur. Phys. J. B, 8 (1999), 525.

3. M. Kardar, G. Parisi, and Y.-C. Zhang, Phys. Rev. Lett. 56 (1986), 889.

4. T. Halpin-Healy and Y.-C. Zhang, Phys. Rep. 254 (1995), 215.

5. M. Lässig, J. Phys. Cond. Mat. 10 (1998), 9905.

6. G. Blatter, M. V. Feigel'man, V. B. Geshkenbein, A. I. Larkin, and V. M. Vinokur, Rev. Mod. Phys. 66 (1994), 1125.

7. J. L. Cardy and S. Ostlund, Phys. Rev. B 25 (1982), 6899.

8. J. Toner and D. P. DiVincenzo, Phys. Rev. B 41 (1990), 632.

9. J.-Ph. Bouchaud, L. F. Cugliandolo, J. Kurchan, and M. Mézard in Spin Glasses and Random Fields, ed. A. P. Young, (World Scientific, Singapore 1997); T. Giarmarchi and P. Le Doussal, ibid.

10. D. S. Fisher and D. A. Huse, Phys. Rev. B 38 (1988), 386; for recent work see M. Palassini and A. P. Young, Phys. Rev. Lett. 83 (1999), 5126.

11. M. Mézard, G. Parisi, and M. A. Virasoro, Spin Glass Theory and Beyond (World Scientific, Singapore 1987); K. Binder and A. P. Young, Rev. Mod. Phys. 58 (1986), 801.

12. R. Lipowsky and M. E. Fisher, Phys. Rev. Lett. 56 (1986), 472 .

13. J. Wuttke and R. Lipowsky, Phys. Rev. B 44 (1991), 13 042 .

14. S. Dietrich in Phase Transitions and Critical Phenomena, edited by C. Domb and J. L. Lebowitz (Academic Press, San Diego 1988), vol. 12; G. Forgacs, R. Lipowsky and Th. M. Nieuwenhuizen in Phase Transitions and Critical Phenomena, edited by C. Domb and J. L. Lebowitz (Academic Press, San Diego 1991), vol. 14.

15. See for recent studies, e.g., S. Scheidl and Y. Dinçer, condmat/0006048; P. Chauve, P. Le Doussal, and K. Wiese, Phys. Rev. Lett. 86 (2001), 1785.

16. L. Balents and D. S. Fisher, Phys. Rev. B 48 (1993), 5949. 17. D. A. Huse and C. L. Henley Phys. Rev. Lett. 54 (1985), 2708.

18. E. T. Seppälä, V. Petäjä, and M. J. Alava, Phys. Rev. E 58 (1998), R5217.

19. D. S. Fisher and D. A. Huse, Phys. Rev. B 43 (1991), 10 728.

20. For the spin glass case see J. Houdayer and O. C. Martin, Phys. Rev. Lett. 81 (1998), 2554.

21. M. Mézard, J. Phys. France 51 (1990), 1831.

22. T. Hwa and D. S. Fisher, Phys. Rev. B 49 (1994), 3136.

23. E. T. Seppälä and M. J. Alava, Phys. Rev. Lett. 84 (2000), 3982 . 
24. E. T. Seppälä, M. J. Alava, and P. M. Duxbury, Phys. Rev

E, in press, e-print: cond-mat/0102318.

25. G. Parisi, J. Phys. France 51 (1990), 1595.

26. B. Derrida, Phys. Rev. B 24 (1981), 2613.

27. J.-P. Bouchaud and M. Mézard, J. Phys. A 30 (1997) 7997.

28. E. J. Gumbel, Statistics of Extremes, (Columbia University Press, New York 1958).

29. Y. Shapir, Phys. Rev. Lett. 66 (1991), 1473.

30. P. Jögi and D. Sornette, Phys. Rev. E 57 (1998), 6936.

31. M. Huang, M. E. Fisher, and R. Lipowsky, Phys. Rev. Ei 39 (1989), 2632.

32. See also J. Galambos, The Asymptotic Theory of Extreme: Order Statistics, (John Wiley \& Sons, New York 1978), sec. tion II.

33. P. M. Duxbury, P. L. Leath, and P. D. Beale, Phys. Rev. E; 36 (1987), 367; P. M. Duxbury, P. L. Leath, and P. D. Beale, Phys. Rev. Lett. 57 (1986), 1052.

34. J. A. Kim, M. A. Moore, and A. J. Bray, Phys. Rev. A 44 (1991), 2345.

35. S. C. Chapman, G. Rowlands, and N. W. Watkins, condmat/0007275.

36. J.-Ph. Bouchaud and A. Georges, Phys. Rev. Lett. 68 (1992), 3908.

37. M. J. Alava and P. M. Duxbury, Phys. Rev. B 54 (1996), 14990.

38. V. I. Räisänen, E. T. Seppälä, M. J. Alava, and P. M. Duxbury, Phys. Rev. Lett. 80 (1998), 329.

39. E. T. Seppälä, M. J. Alava, and P. M. Duxbury, Phys. Rev. E 63 (2001), 036126.

40. M. Alava, P. Duxbury, C. Moukarzel, and H. Rieger, in Phase Transitions and Critical Phenomena, edited by C. Domb and J. L. Lebowitz (Academic Press, San Diego 2001), vol. 18.

41. J. C. Picard and H. D. Ratliff, Networks 5 (1975), 357.

42. L. R. Ford and D. R. Fulkerson, Flows in Networks, (Princeton University Press, Princeton 1962) see also any standard book on graph theory and flow problems.

43. A. V. Goldberg and R. E. Tarjan, J. Assoc. Comput. Mach. 35 (1988), 921.

44. Note that one can also use the level crossing effect to compute the crossing points exactly, as noticed by Anglès d'Auriac and Sourlas [49. This allows to use the residual graph of the original groundstate for the further computations, and makes the solution faster 40].

45. S. Roux, A. Hansen, and E. Guyon, J. Phys. (Paris) 48 (1987), 2125.

46. M. Aizenman and J. Wehr, Phys. Rev. Lett. 62 (1989), 2503.

47. A.-L. Barabási and H. E. Stanley, Fractal concepts in surface growth, (Cambridge University Press, Cambridge, U.K. 1995).

48. See an example of effects of boundary conditions: M. Prähofer and H. Spohn, Phys. Rev. Lett. 84 (2000), 4882.

49. J. C. Anglès d'Auriac and N. Sourlas, Europhys. Lett. 39 (1997), 473.

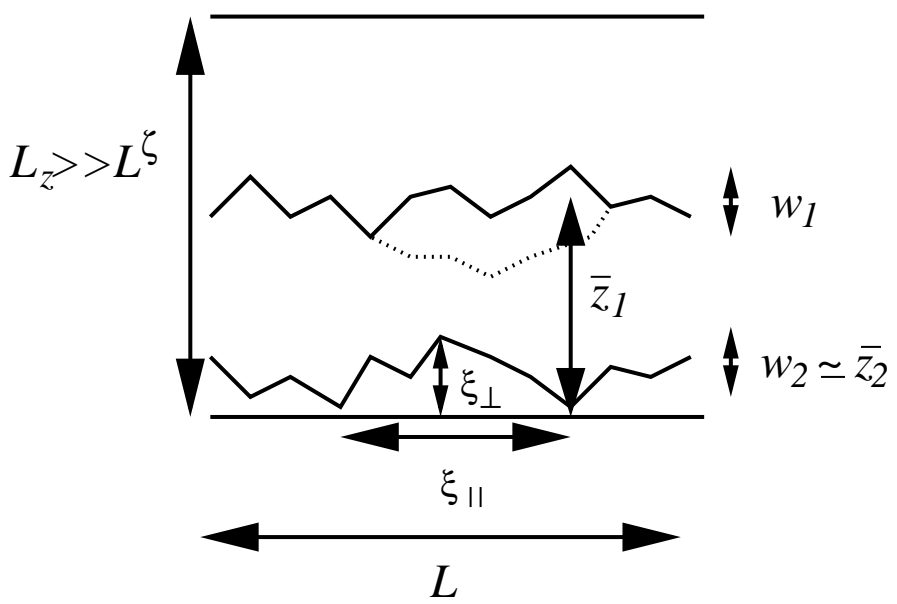

Fig. 1. A schematic figure of two interfaces in a system of parallel length $L$ and perpendicular one $L_{z}>L^{\zeta}$ at heights $\bar{z}_{1}$ and $\bar{z}_{2} \simeq w_{2}$, with the corresponding roughness values $w_{1}$ and $w_{2} . \xi_{\perp}$ denotes the transverse correlation length of a part of the interface of length $\xi_{\|}$. See the text. A droplet is seen in the interface of height $\bar{z}_{1}$ as a dotted line. 
E. T. Seppälä and M. J. Alava: Energy landscapes, lowest gaps, and susceptibility of elastic manifolds at $T=0$
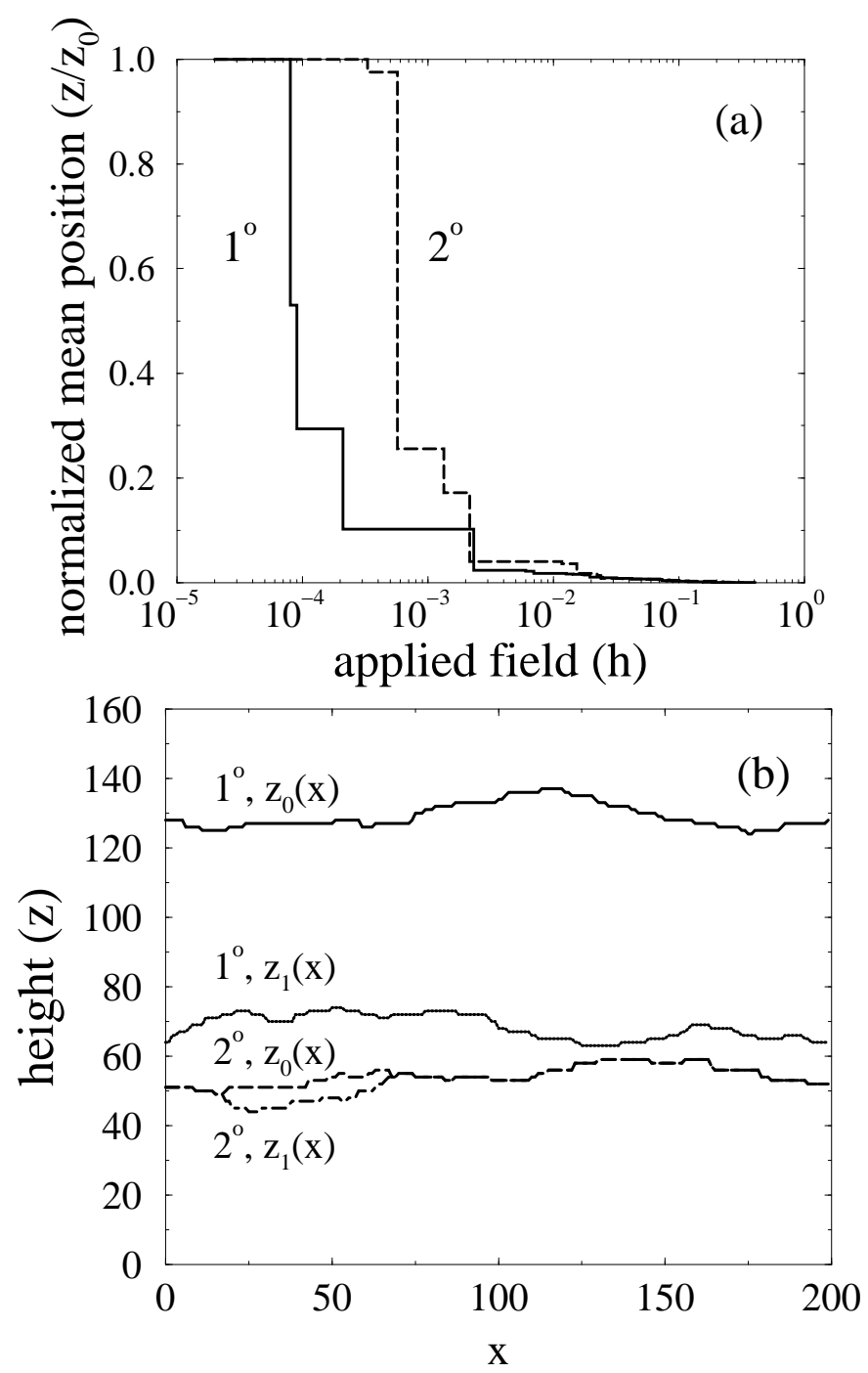

Fig. 2. (a) Examples of two realizations of changes in mean heights $\bar{z}$ of interfaces normalized by their original (global minimum) positions $\bar{z}_{0}$ vs. applied field $h$ for $(1+1)$ dimensional systems. The change in the external field is done in steps of $\Delta h=10^{-5}$. Note the large jumps in both cases. $L^{2}=200^{2}$. $J_{i j, z} \in[0-1]$ uniform distribution and $J_{i j, x}=0.5$ (random bond disorder). (b) The expected scenarios (droplet formation, jump to the lower edge of the system) before and after the first moves from global minima $z_{0}(x)$ to $z_{1}(x)$.

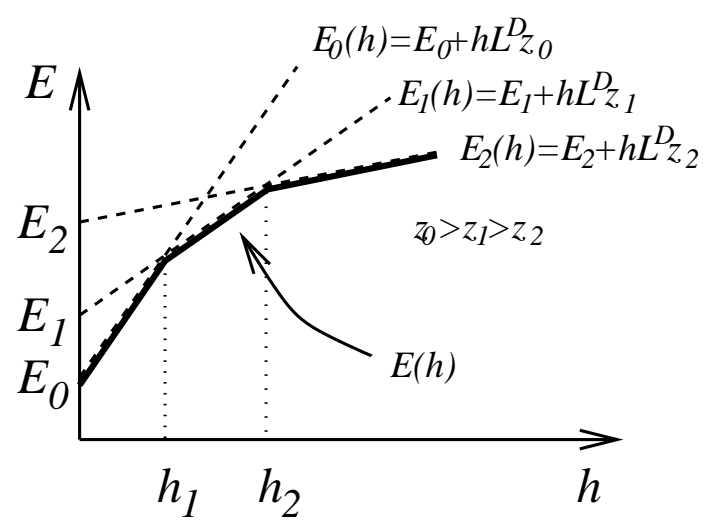

Fig. 3. The level-crossing phenomenon for interfaces in random systems in the presence of an external field. Originally the interface lies at height $z_{0}$ and has an energy $E_{0}$. When the field is applied its energy increases linearly and at $h_{1}$ the interfaces jumps to $z_{1}<z_{0}$, with the energy $E_{1}\left(h_{1}\right)=E_{1}+h_{1} L^{D} z_{1}$, where $E_{1}$ is the energy of the interface at $z_{1}$ without the field. Similar behavior takes place at $h_{n}, n=2,3, \ldots$, when the interface moves from $z_{n-1}$ to $z_{n}<z_{n-1}$. The thick line represents the minimum energy of the interface $E(h)$ as a function of the field $h$, with discontinuities of the derivative at $h_{n}$.

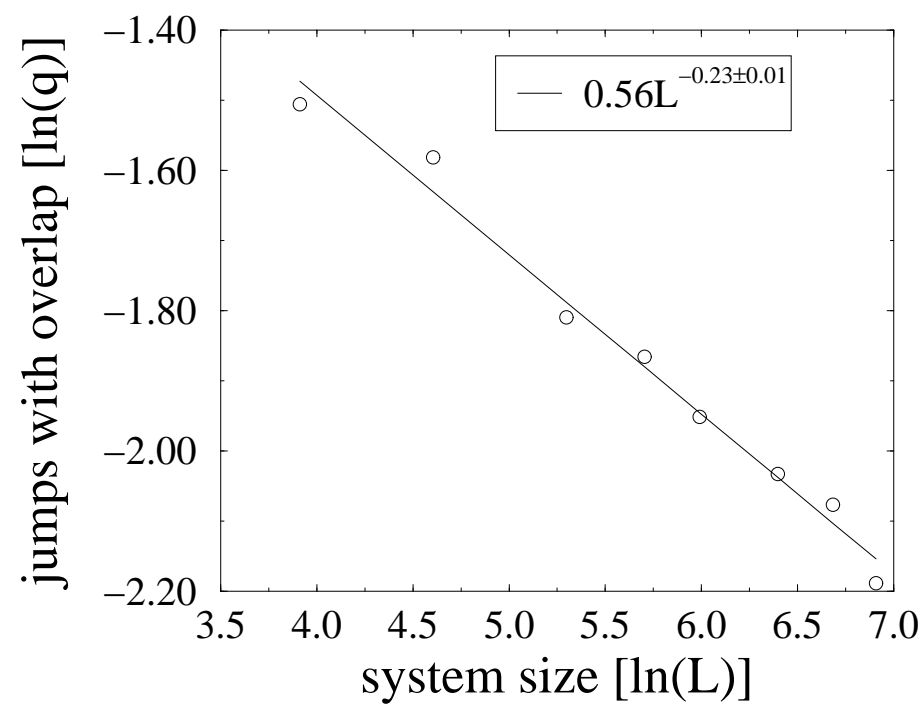

Fig. 4. The fraction of jumps of interfaces with initial global minimum in an arbitrary position that have an overlap between interfaces before and after jump $q$ as function of the system sizes $L$. The system sizes are $L^{2}=50^{2}-1000^{2}$ and the number of realizations $N=3000-5000$. The line is a least-squares fit to the data with the power-law behavior $L^{-0.23 \pm 0.01}$. 
(a)
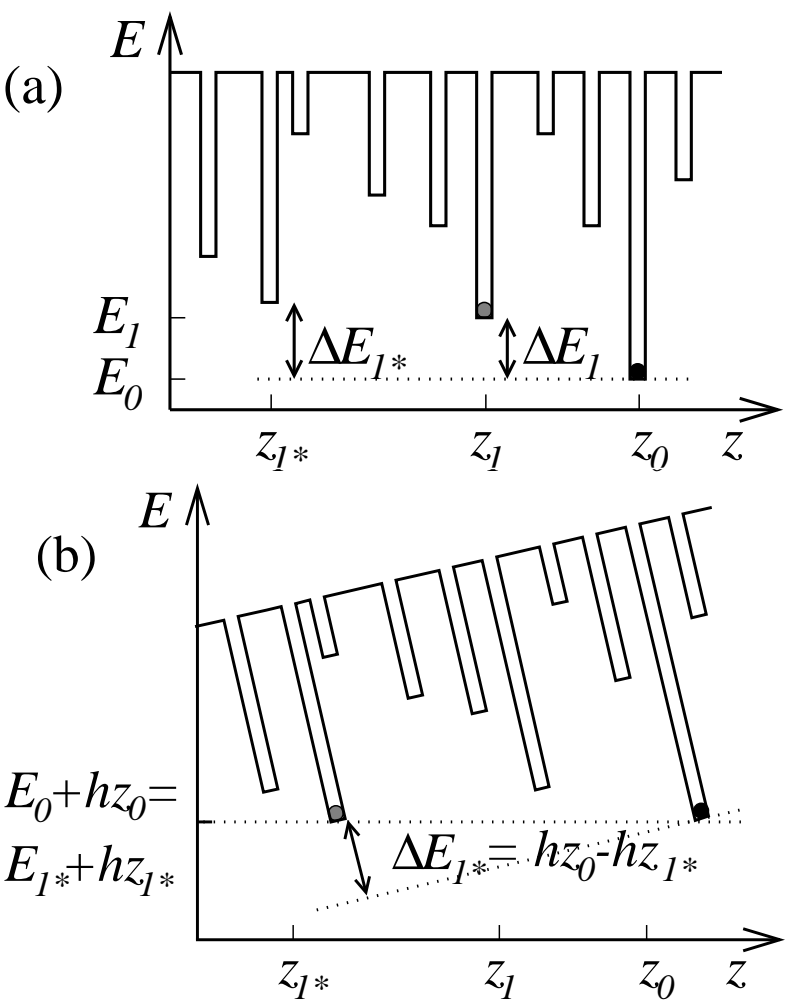

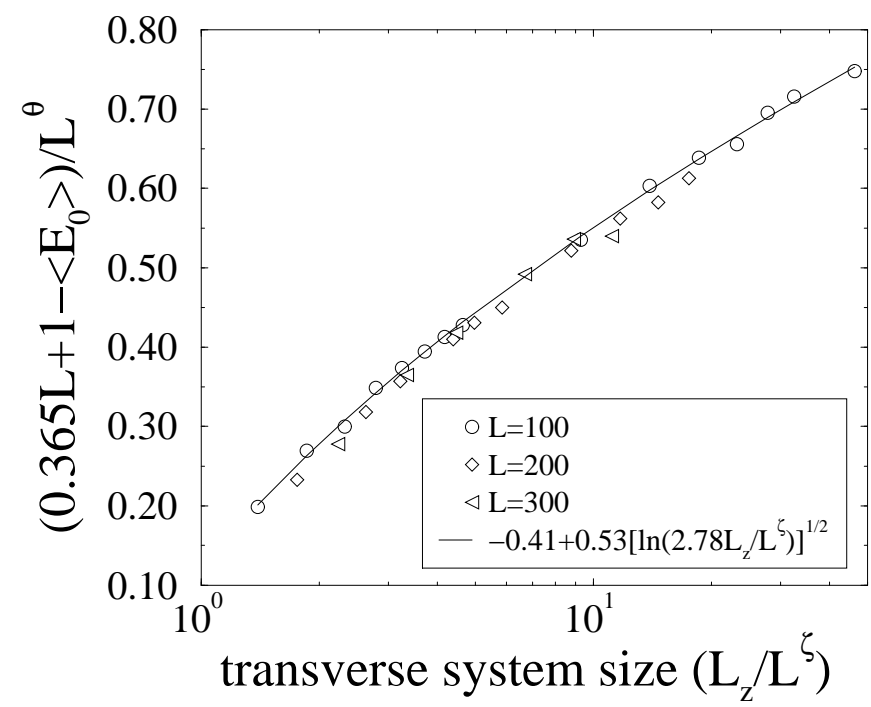

Fig. 6. The scaling of the ground state energy, $\left(\langle E\rangle-\left\langle E_{0}\right\rangle\right) / L^{\theta}$ vs. $L_{z} / L^{\zeta}$, see Eq. (25) and the text, for the system sizes $L=100,200$, and 300 , each with $\bar{z}_{0} / L_{z} \simeq$ const. $\theta=1 / 3$, $\zeta=2 / 3$. The line $-0.41+0.53\left[\ln \left(2.78 L_{z} / L^{\zeta}\right)\right]^{1 / 2}$ is a guide to the eye. The number of realizations ranges from $N=500$ for $L=300, L_{z}=500$ to $N=2000$ for $L=200, L_{z}=600$.

Fig. 5. (a) A simplified view of the minima in the energy landscape of a random system. $\Delta E_{1}=E_{1}-E_{0}$ is the energy gap between the ground state at $z_{0}$, denoted with a black circle, and the second lowest minimum at $z_{1}$ denoted with a gray circle. $\Delta E_{1 *}=E_{1 *}-E_{0}$ is the energy gap (normalized with $L^{D}$ ) between the ground state and the minimum at $z_{1^{*}}$. (b) The view of the minima in the random system when the field is applied. At $h_{1}$ the interface moves from $z_{0}$ to $z_{1^{*}}$, indicated with a gray circle, since the energy difference $\Delta E_{1^{*}}=E_{1^{*}}-$ $E_{0}=h_{1} z_{0}-h_{1} z_{1^{*}}=0$ while all the other $\Delta E$ 's are greater. However, often $E_{1 *}$ and $E_{1}$ coincide. 

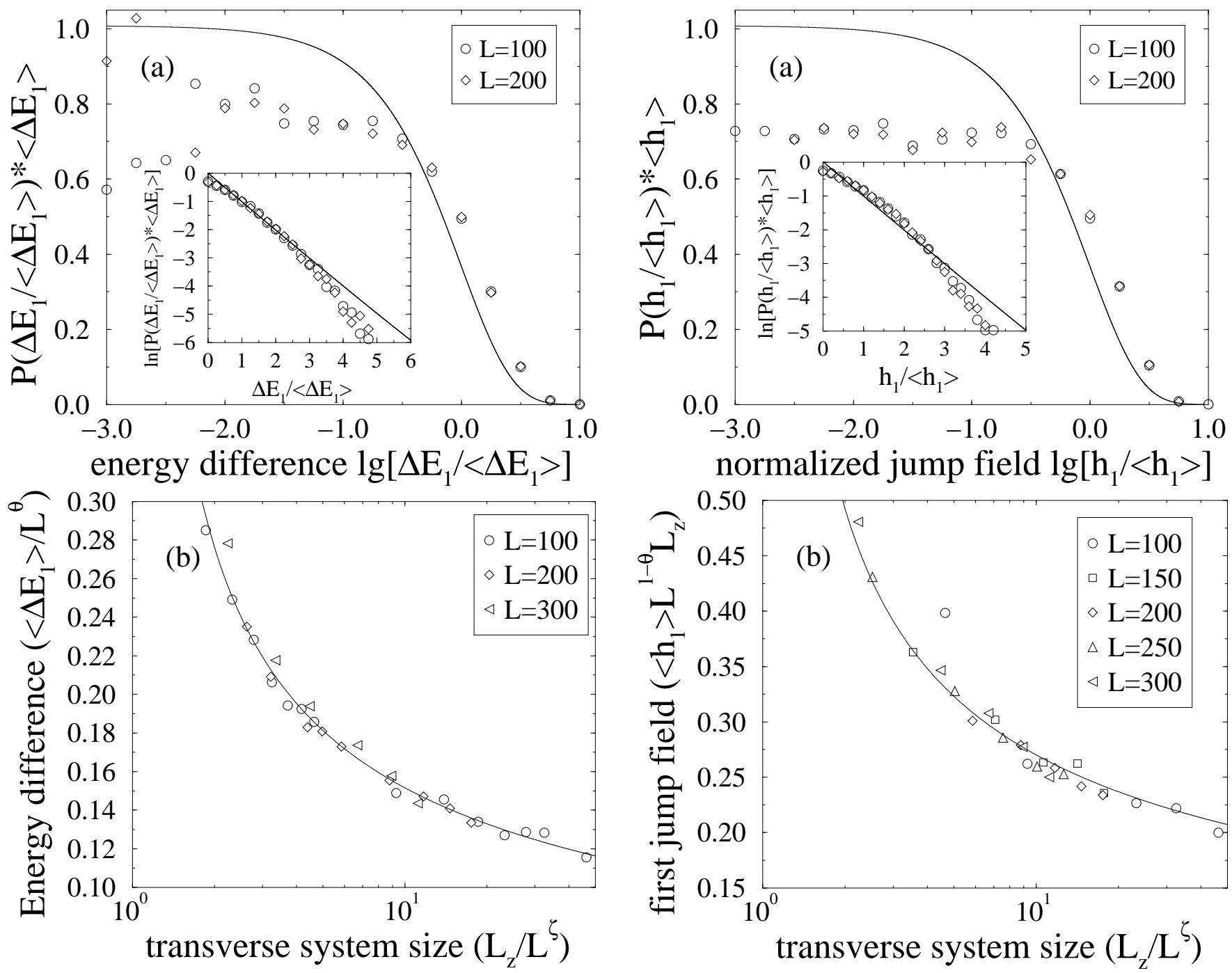

Fig. 7. (a) The scaling function of the probability distribution $\hat{P}\left(\Delta E_{1} /\left\langle\Delta E_{1}\right\rangle\right) \times\left\langle\Delta E_{1}\right\rangle$ for the energy gaps $\Delta E_{1}$ of the lowest two minima normalized by their disorder-average $\left\langle\Delta E_{1}\right\rangle$ in a (10-base) semilog-scale for the system sizes $L \times L_{z}=L^{2}=100^{2}$ and $200^{2}$. The inset shows the tails in the natural-log-scale. The initial global minimum position $\bar{z}_{0} / L_{z} \simeq$ const for all $L$. The number of realizations $N=2 \times 10^{4}$ for both system sizes. The lines in the figure and in the inset are $\exp (-x)$. (b) The scaling function $f(y)$ of the scaled disorder-average of the energy gap $\left\langle\Delta E_{1}\right\rangle / L^{\theta}$ as a function of scaled transverse system size $L_{z} / L^{\zeta}$ for the system sizes $L=100,200$, and 300, each with $\bar{z}_{0} / L_{z} \simeq$ const. $\theta=1 / 3, \zeta=2 / 3$. The line $f(y)=0.23 \ln (y)^{-1 / 2}$ is a guide to the eye. The number of realizations ranges from $N=500$ for $L=300, L_{z}=500$ to $N=2000$ for $L=200$, $L_{z}=600$. Only the non-overlapping interfaces are considered. The fraction of overlapping ones is vanishingly small.

Fig. 8. (a) The scaling function of the probability distribution $P\left(h_{1} /\left\langle h_{1}\right\rangle\right) \times\left\langle h_{1}\right\rangle$ for the first jump field values $h_{1}$ normalized by their disorder-average $\left\langle h_{1}\right\rangle$ in a (10-base) semilog-scale for the system sizes $L \times L_{z}=L^{2}=100^{2}$ and $200^{2}$. The inset shows the tails in the natural-log-scale. The initial global minimum position $\bar{z}_{0} / L_{z} \simeq$ const for all $L$. The number of realizations $N=2 \times 10^{4}$ for both system sizes. The line is the analytic result from Eq. (13) with a uniform distribution $\hat{P}(x)$ and $N_{z}=20$. (b) The scaling function $f(y)$ of the scaled disorder-average of the jump field $\left\langle h_{1}\right\rangle L^{1-\theta} L_{z}$ as a function of scaled transverse system size $L_{z} / L^{\zeta}$ for the system sizes $L=100,150,200,250$ and 300 , each with $\bar{z}_{0} / L_{z} \simeq$ const. $\theta=1 / 3, \zeta=2 / 3$. The line $f(y)=0.41 \ln (y)^{-1 / 2}$ is a guide to the eye. The number of realizations ranges from $N=500$ for $L=300, L_{z}=500$ to $N=2600$ for $L=200, L_{z}=600$. Only the non-overlapping jumps are considered, again. 


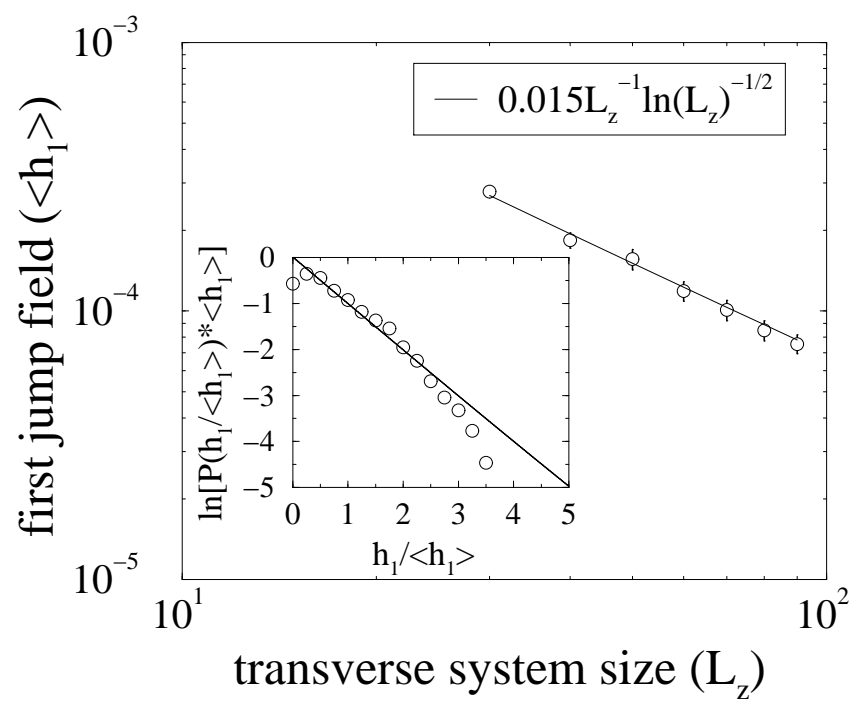

Fig. 9. The disorder-average of the first jump field $\left\langle h_{1}\right\rangle$ as a function of transverse system size $L_{z}$ for the $(2+1)$ dimensional interface. The disorder is random bond type, with $\delta J_{i j} / J_{0}=1$, the number of realizations for each system size $N \geq 100$, and the system size $L_{x}=L_{y}=50$. The initial global minimum position $\bar{z}_{0} / L_{z} \simeq$ const. The line $0.015 L_{z}^{-1}\left[\ln \left(L_{z}\right)\right]^{-1 / 2}$ is a guide to the eye. The inset shows the tail of the scaling function of the probability distribution $P\left(h_{1} /\left\langle h_{1}\right\rangle\right) \times\left\langle h_{1}\right\rangle$ for the first jump field values $h_{1}$ normalized by the disorder-average $\left\langle h_{1}\right\rangle$ in a natural-log-scale for the system size $L^{3}=50^{3}$. The initial global minimum position $\bar{z}_{0} / L_{z} \simeq$ const. The disorder is dilution type with $p=0.5$ and the first non-overlapping jump is considered. The number of realizations $N>3000$. The line is the same as in the inset of Fig. 8(a).
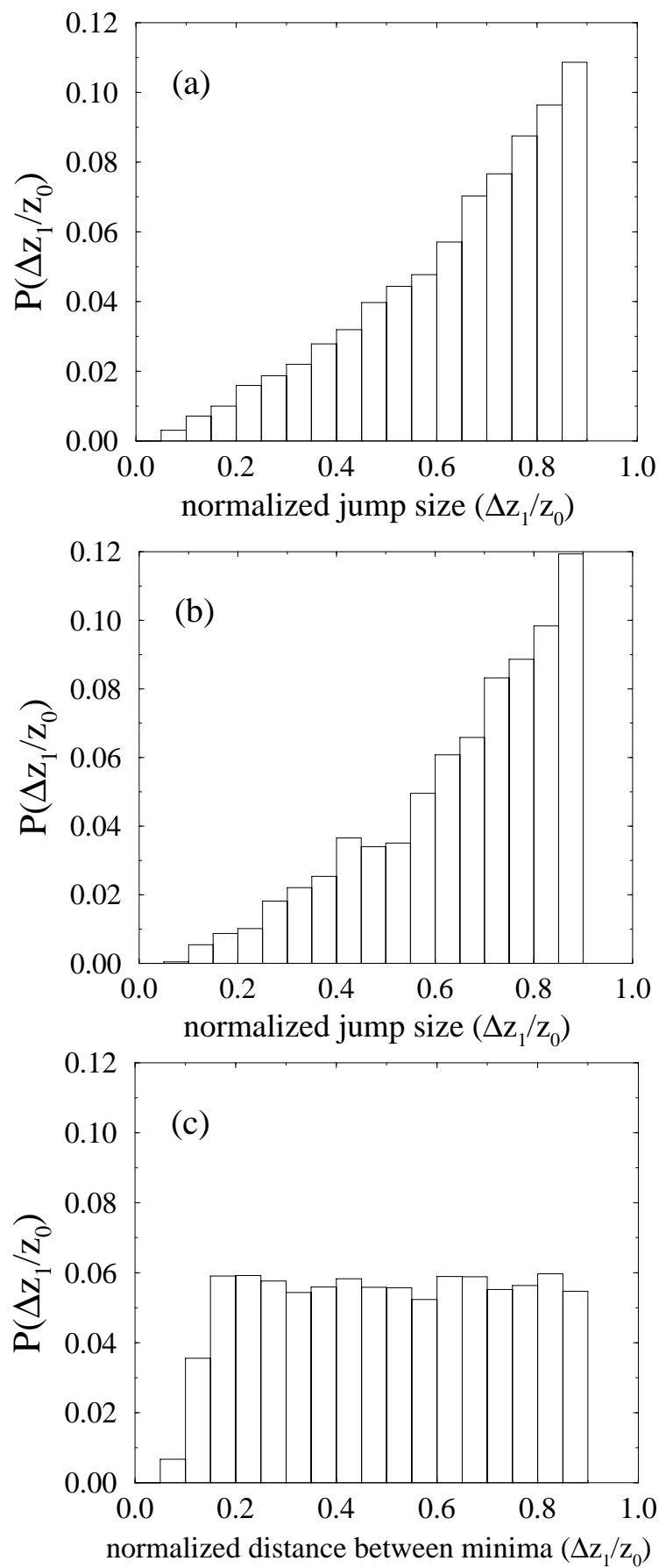

Fig. 10. (a) The histogram $P\left(\Delta z_{1} / z_{0}\right)$ of the jump size $\Delta z_{1}$ of the first jump normalized with the initial position $z_{0}$ (the initial global minimum position $\bar{z}_{0} \simeq$ const) for $(1+1)$ dimensional interface. The number of realizations $N=2 \times 10^{4}$, the system size $L^{2}=100^{2}$, and the random configurations are the same as in Fig. 8(a). (b) $P\left(\Delta z_{1} / z_{0}\right)$ for $(2+1)$ dimensional interface The number of realizations $N \simeq 3000$ and the random configurations are the same as for the data in Fig. 9 inset. The system size $L^{3}=50^{3}$ and disorder is dilution type with $p=0.5$. The initial global minimum position $\bar{z}_{0} \simeq$ const. (c) $P\left(\Delta z_{1} / z_{0}\right)$ for $(1+1)$ dimensional interface without a field, $L^{2}=100^{2}$, the number of realizations $N=2 \times 10^{4}$. The random configurations are the same as in Fig. 7 (a) and the initial global minimum position $\bar{z}_{0} \simeq$ const. Note in all figures the repulsion induced behavior at the both ends: for values close to zero due to neglect of the overlapping interfaces and for values close to unity due to wall repulsion. 
E. T. Seppälä and M. J. Alava: Energy landscapes, lowest gaps, and susceptibility of elastic manifolds at $T=0$
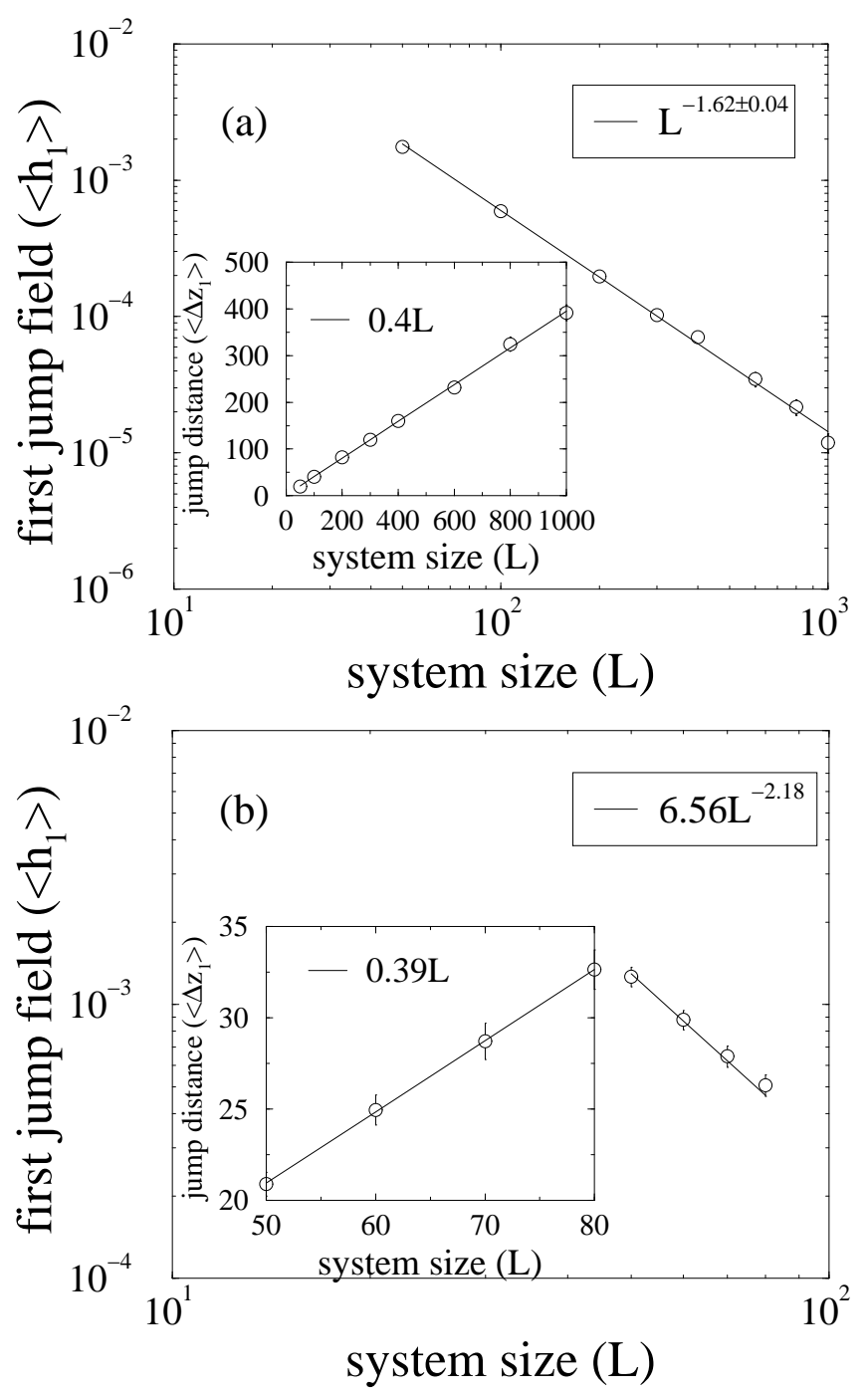

Fig. 11. (a) Finite size scaling of the average first jump field $\left\langle h_{1}\right\rangle$ for DP's. The line is the least squares fit to data. The scaling argument gives $\alpha=5 / 3$. The inset shows the average jump distance $\left\langle\Delta z_{1}\right\rangle$ at the corresponding field $h_{1}$ with a linear fit to data. \langle\rangle is the disorder-average over $N=1000$ realizations for the system sizes $L \times L_{z}=L^{2}=50^{2}$ and $100^{2}, N=500$ for $L^{2}=200^{2}-400^{2}$, and $N=200$ for $L^{2}=600^{2}-1000^{2}$. The disorder is of random bond type. No overlaps included. (b) Finite size scaling of the average first jump field $\left\langle h_{1}\right\rangle$ for $(2+1)$ dimensional manifold. The line is a guide to the eye with a slope $\alpha \simeq 2.18$. The inset shows the average jump distance $\left\langle\Delta z_{1}\right\rangle$ at the corresponding field $h_{1}$ with a linear fit to data. \langle\rangle is the disorder-average over $N \simeq 250$ realizations for each system size. The disorder is dilution type with $p=0.5$ and the first non-overlapping jump is considered (usually two small-scale adjustments take place before the jump).

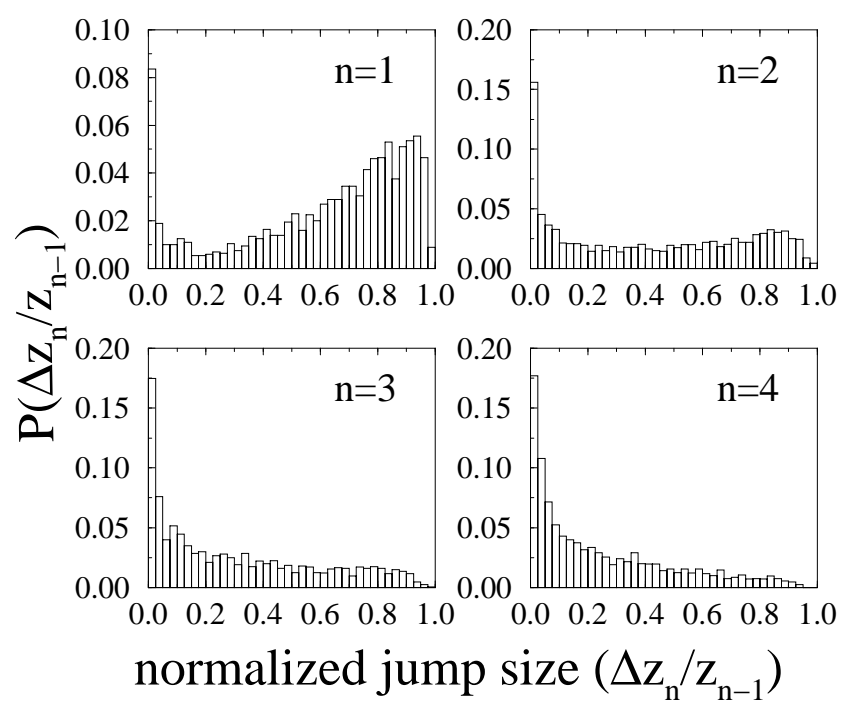

Fig. 12. Evolution of the histogram of the jump sizes $\Delta z_{n} / z_{n-1}$ normalized with its previous position for $n=$ $1,2,3,4$. The system size is $L^{2}=100^{2}$, the number of realizations $N=2000$. The overlapping jumps are included. 

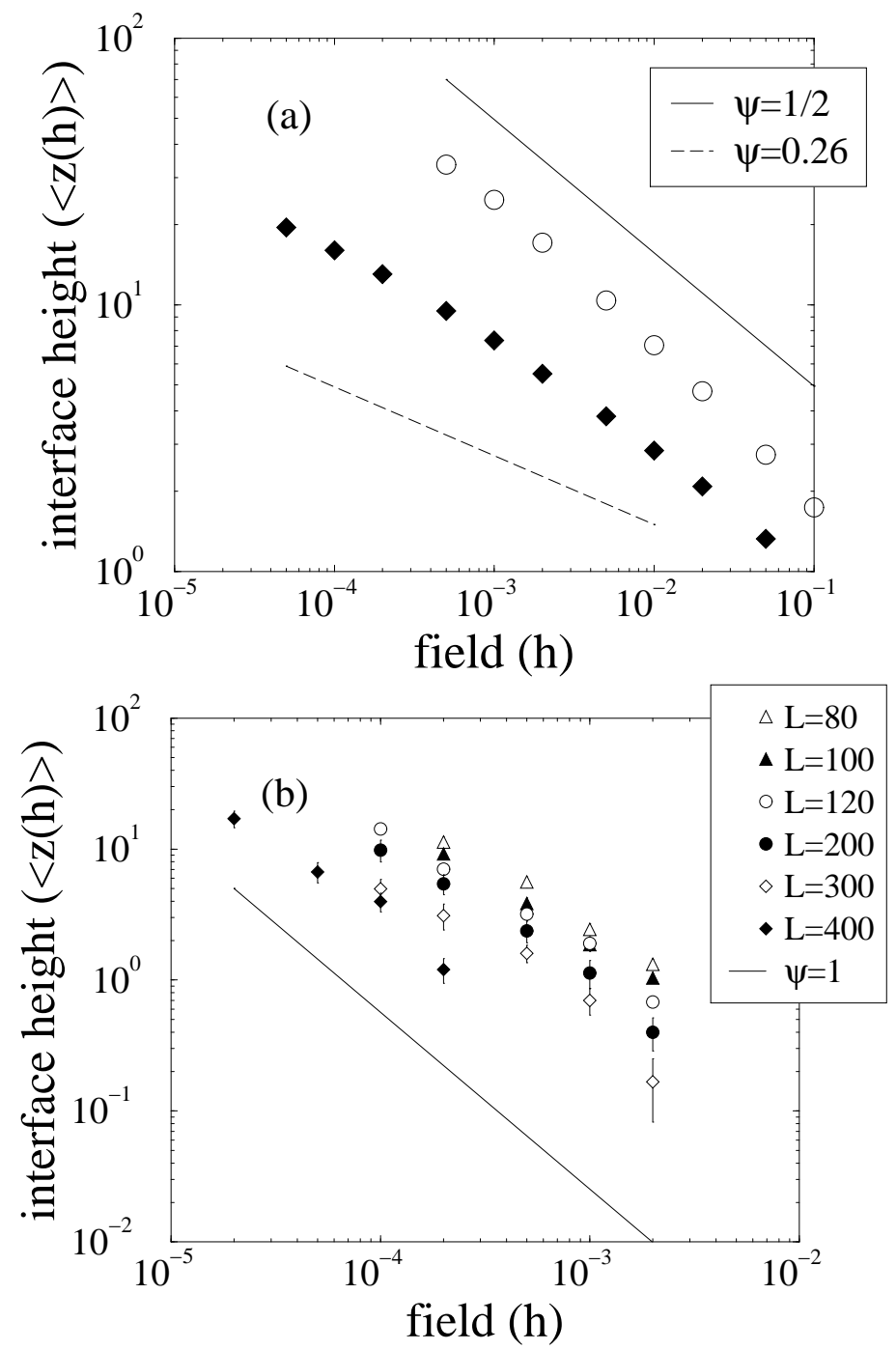

Fig. 13. (a) The average interface mean height $\langle\bar{z}(h)\rangle$, i.e., the thickness of wetting layer, as a function of the external field $h$ for one dimensional directed polymer, open circles. The system size is $L=3000, L_{z}=100$ and the number of realizations $N=$ 1000. The disorder is dilution type with $p=0.55$. The filled diamonds denote $(2+1)$ dimensional interfaces. The system size is $L_{x}=L_{y}=300, L_{z}=50$ and the number of realizations $N=30$. The disorder is dilution type with $p=0.30$. The solid line is a guide to the eye with a slope $\psi=1 / 2$ and the dashed line has a slope $\psi=0.26$. (b) $\langle\bar{z}(h)\rangle$ vs. $h$ for various system sizes $L_{x}=L_{y}=L=80,100,120,200,300$, and 400 in the flat regime. The disorder is dilution type with $p=0.95$. $L_{z}=50$ for each system. The number of random configurations is $N=100-$ -200 for $L=80-120$, and $N=30$ for $L=200-400$. The line is a guide to the eye with a slope $\psi=1$.
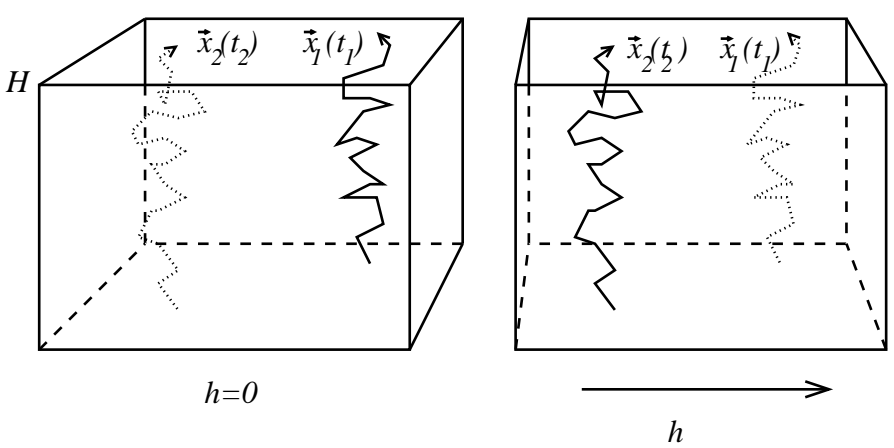

Fig. 14. The relation between directed polymers and growing interfaces. Two directed polymers in independent valleys equal the fastest arrival time $t_{1}$ at $\mathbf{x}_{1}$, solid line, and the second fastest at $\mathbf{x}_{2}$ with time $t_{2}$, dotted line, of a KPZ interface to a fixed height $H$, when the external field $h=0$. In the right hand side figure an external field is added, which increases the growth time depending on the position in the direction of the arrow and the polymer at $\mathbf{x}_{2}$ becomes the one corresponding to the fastest time to reach $H$. 